\title{
Holistic principle for risk aggregation and capital allocation
}

\author{
Wing Fung Chong ${ }^{1,2} \mathbb{D} \cdot$ Runhuan Feng $^{1} \cdot$ Longhao Jin $^{1}$
}

Accepted: 5 February 2021

(c) The Author(s) 2021

\begin{abstract}
Risk aggregation and capital allocation are of paramount importance in business, as they play critical roles in pricing, risk management, project financing, performance management, regulatory supervision, etc. The state-of-the-art practice often includes two steps: (i) determine standalone capital requirements for individual business lines and aggregate them at a corporate level; and (ii) allocate the total capital back to individual lines of business or at more granular levels. There are three pitfalls with such a practice, namely, lack of consistency, negligence of cost of capital, and disentanglement of allocated capitals from standalone capitals. In this paper, we introduce a holistic approach that aims to strike a balance of optimality by taking into account competing interests of various stakeholders and conflicting priorities in a corporate hierarchy. While unconventional in its objective, the new approach results in an allocation of diversification benefit, which conforms to the diversification strategy of many risk management frameworks including regulatory capital and economic capital. The holistic capital setting and allocation principle provides a remedy to aforementioned problems with the existing two-step industry practice.
\end{abstract}

Keywords Risk management · Risk aggregation · Capital allocation · Pareto optimality · Diversification benefit

\section{Introduction}

Due to the modern development in the financial and insurance markets, as well as the increasing complexity of their business models, any financial service organization is exposed to an array of risk factors. Sufficient capitals are critical under adverse changes in these risk factors to provide the buffer for absorbing any unexpected losses. Capital requirements are also fundamental tools for regulators to safeguard the interest of the public. For example, regulators around the world have already taken significant measures to tighten regulations, particularly on the solvency capital requirement, since the 2008 financial crisis.

$凶$ Wing Fung Chong

wfchong@illinois.edu

1273 Altgeld Hall, MC-382, Department of Mathematics, University of Illinois at Urbana-Champaign, 1409 West Green Street, Urbana, Illinois 61801, USA

2 Department of Statistics, University of Illinois at Urbana-Champaign, Champaign, Illinois, USA 
The purpose of this paper is to re-examine the fundamental philosophy behind the stateof-the-art capital practice and regulation, and to uncover several major pitfalls of the current system. As a remedy, we propose a holistic approach to address the competing goals in a corporate hierarchy and to reach an optimal capital solution for an entire corporate and its components.

\subsection{Risk aggregation and capital allocation}

To understand the current practice on risk aggregation and capital allocation, we point to Fig. 1 which depicts a typical hierarchy of a financial corporate.

Risk aggregation refers to the process of consolidating risk exposures from the bottom level (e.g. individual risks, contracts, lines of business, business units, subsidiaries) of a business hierarchy to a risk profile at the top level (e.g. corporate group). A common approach to determine capital is to estimate the potential loss/profit at the bottom level and apply certain risk measure to determine their standalone capitals. The total capital at the top level is the aggregation of standalone capitals from the bottom level. To reflect the diversification benefit, aggregate capital is often set to be less than the sum of standalone capitals. The most wellknown example is the variance-covariance approach, which has been further studied in the literature; see, for example, Dhaene et al. (2005), Pfeifer and Strassburger (2008), Filipović (2009), and Arbenz et al. (2012). There are several other approaches of risk aggregation in the literature, such as modular approaches in Perli and Nayda (2004) and Bølviken and Guillen (2017), scenario aggregation approaches, model uncertainty approaches in Embrechts et al. (2013), Bernard et al. (2014), Sarabia et al. (2016), and Di Lascio et al. (2018), as well as moment-based approaches in Cossette et al. (2016), Miles et al. (2020), and Furman et al. (2020).

Capital allocation refers to the process of dividing overall capital to more granular levels of the business. The decision-making process behind allocation can be quite complex in practice, taking into account risk management policies, regulatory requirements, internal performance measures, etc. Euler (or marginal; or gradient) allocation principle is a common approach in practice; see, such as, Tasche (1999). Other common approaches

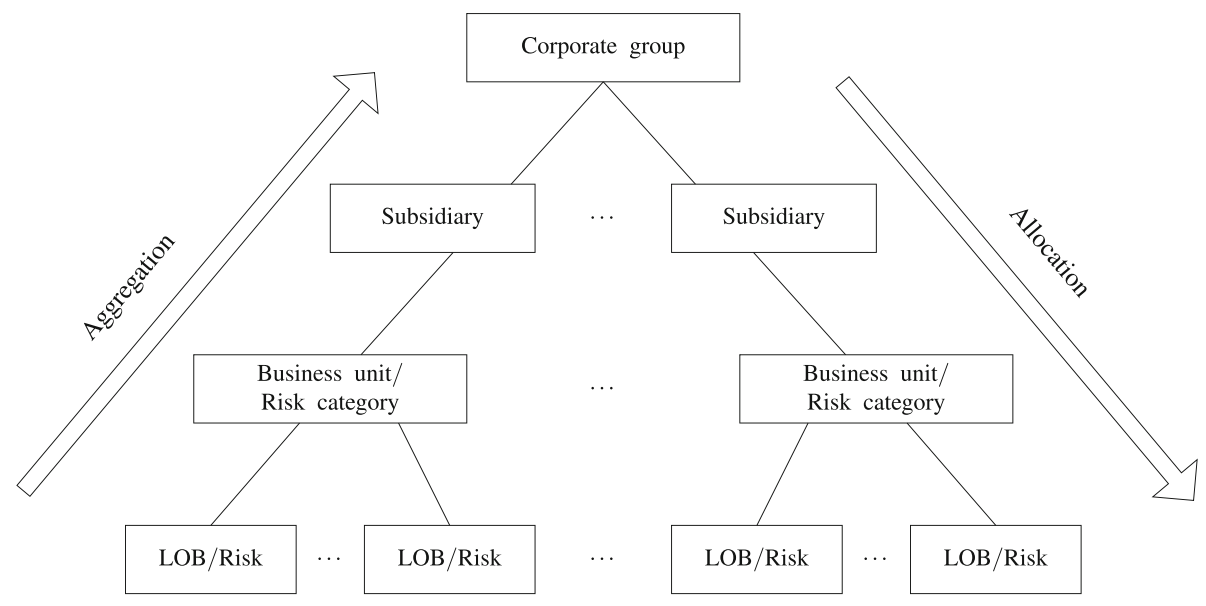

Fig. 1 Corporate hierarchy for risk aggregation and capital allocation 
include axiomatic approaches in Denault (2001), Kalkbrener (2005), and Buch and Dorfleitner (2008), game-theoretic applications in Tsanakas and Barnett (2003), Powers (2007), and Boonen et al. (2017), as well as optimization in Laeven and Goovaerts (2004), Zaks et al. (2006), Buch et al. (2011), Dhaene et al. (2012), Kang and Poshakwale (2019), Baione et al. (2020), and Chen et al. (2020).

The list of papers above is far from comprehensive. Detailed accounts can be found in Bauer and Zanjani (2014), Bauer and Zanjani (2016), Asimit et al. (2019), Boonen (2019), Gómez and Tang (2020), and the references therein. For market practices on risk aggregation and capital allocation in various industries, see Corrigan et al. (2009), McNeil et al. (2015), and Feng (2018). The variance-covariance approach and the Euler allocation principle shall be reviewed in Sect. 3.3 when they are compared with the holistic approach proposed in this paper.

\subsection{Quantitative framework}

This section sets the quantitative framework for risk aggregation and capital allocation throughout this paper. For brevity, we shall only consider in this paper two levels of the corporate hierarchy, with either individual risks or lines of business (LOBs/risks) at the bottom, and the corporate management at the top. Similar results can be extended to corporate hierarchy with more tiers, as depicted in Fig. 1.

Let $(\Omega, \mathcal{F}, \mathbb{P})$ be a rich enough underlying probability space. Consider a corporate which holds $n$ individual LOBs/risks. In a static setting, a capital allocation principle is a general mapping $\mathcal{C}: \mathcal{X}_{1} \times \mathcal{X}_{2} \times \cdots \times \mathcal{X}_{n} \rightarrow \mathbb{R}^{n}$, where $\mathcal{X}_{i}$, for $i=1,2, \ldots, n$, denotes the collection of random variables, defined on the probability space, representing the $i$ th LOB/risk; the allocation principle maps $\left(X_{1}, X_{2}, \ldots, X_{n}\right) \in \mathcal{X}_{1} \times \mathcal{X}_{2} \times \cdots \times \mathcal{X}_{n}$ to $\mathcal{C}\left(X_{1}, X_{2}, \ldots, X_{n}\right)=$ $\left(K_{1}, K_{2}, \ldots, K_{n}\right) \in \mathbb{R}^{n}$, where $K_{i}$, for $i=1,2, \ldots, n$, represents the allocated capital to the $i$ th LOB/risk. In the remaining of this paper, the domain $\mathcal{X}_{1} \times \mathcal{X}_{2} \times \cdots \times \mathcal{X}_{n}$ and the range $\mathbb{R}^{n}$ are usually dropped for clear exposition.

For instance, without taking into account the potential diversification benefit among the LOBs/risks, the straightforward principle of the allocated capital $K_{i}$, for $i=1,2, \ldots, n$, is given by $\rho_{i}\left(X_{i}\right)$, for some risk measure $\rho_{i}$. The state-of-the-art two-step procedure, risk aggregation and capital allocation, is depicted in Fig. 2, when the potential diversification benefit is taken into consideration via a general mapping $G$ from the random vector $\left(X_{1}, X_{2}, \ldots, X_{n}\right)$ to a constant $K$. Herein, the constant $K$ represents the aggregate capital at the corporate level; by the definition of capital allocation for distributing the aggregate capital to LOBs/risks at the lower level, $K=K_{1}+K_{2}+\cdots+K_{n}$.

\subsection{Criticisms and contributions}

While it may seem natural to treat risk aggregation and capital allocation sequentially in two separate steps, such a practice came to existence for historic reasons with the development of capital regulation. This paper challenges this conventional two-step procedure, and points out its three pitfalls: (i) lack of consistency in risk aggregation and capital allocation, (ii) omission of opportunity cost of capital, and (iii) disconnection of allocated capital, by risk aggregation and capital allocation, from standalone capital requirements.

Firstly, model assumptions for risk aggregation are often incompatible with those for capital allocation. Simply take for example the common practice of the variance-covariance approach for risk aggregation together with the Euler allocation principle for capital allo- 


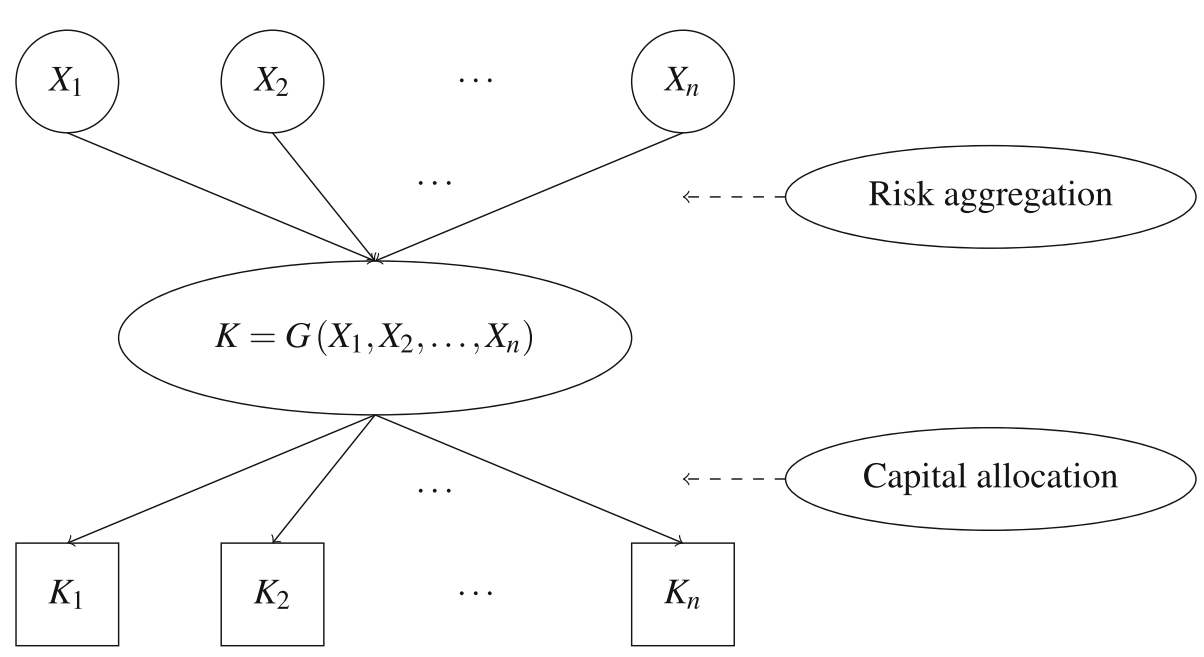

Fig. 2 Two-step procedure-risk aggregation and capital allocation

cation. Assume that the risk measure $\rho$ is given by either the Value-at-Risk (VaR) or the Tail Value-at-Risk (TVaR). Recall that, in the variance-covariance approach, the aggregate capital $K$ might not match the risk measurement of the aggregate loss $\rho(S)$; see also Sect. 3.3.1 below. However, the aggregate capital must equal to the risk measurement of the aggregate loss, in order to adopt the Euler allocation principle. Therefore, to ensure the variance-covariance approach and the Euler allocation principle being compatible, a sufficient condition is that all losses $X_{1}, X_{2}, \ldots, X_{n}$ are multivariate normally distributed. Yet there are many empirical studies showing that normality assumption is often far from reality.

Secondly, within each LOB/risk, or even the corporate level in the corporate hierarchy, there are often competing management goals. On one hand, a prudent risk manager may prefer as much capital as possible in order for the corporate to absorb severe losses under adverse economic conditions; on the other hand, it is undesirable to hold excessive capital as it is kept in liquid assets and has lower financial returns than other assets. Moreover, the financial impact of over-reserving is well documented in the insurance literature, see, for example, Anderson and Thompson (1971). Therefore, it is important to develop a sensible mechanism to strike a balance between the competing capital management and risk management goals. In fact, such a delicate balancing act takes place implicitly when the VaR is used to determine the allocated capital. To illustrate this, let $Y$ be a loss, either individual or aggregate, and the allocated capital to this loss $Y$ is given by $\operatorname{VaR}_{\alpha}(Y)$, where $\alpha \in[0,1]$ is a confidence level. It is well-known that $\operatorname{VaR}_{1-r}(Y)$, where $r=1-\alpha$, is indeed the minimizer of the following optimization problem:

$$
\min _{K \in \mathbb{R}} r K+\mathbb{E}\left[(Y-K)_{+}\right] .
$$

The first term is attributable to capital management objective, as it represents the cost of capital at the borrowing interest rate $r$ and penalizes excessive capital holding. The second term illustrates the risk management objective to minimize the expected shortfall beyond the allocated capital. The rate $r$ also measures the relative importance of capital management objective compared with the risk management objective. When $r=0$, capital management objective is ignored, and the decision is solely based on risk management objective, which is to hold as capital the largest possible value of loss, i.e., $\operatorname{VaR}_{1}(Y)$; when $r$ rises, capital 


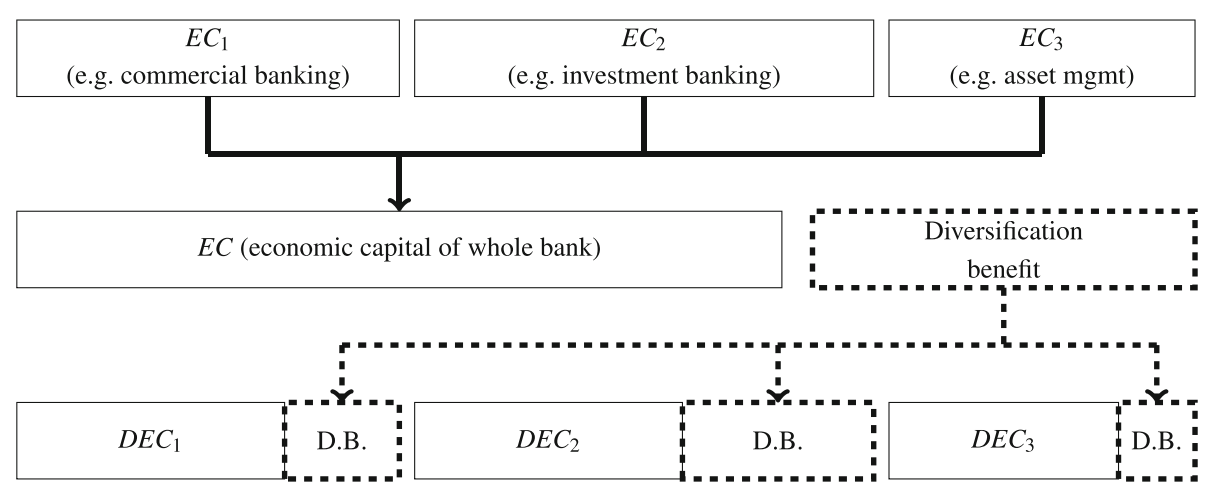

Fig. 3 Diversification benefit and diversified economic capital

management exerts increasing influence on capital setting, pushing down the required capital. When $r=1$, the optimal capital becomes the smallest possible value of loss, i.e., $\operatorname{VaR}_{0}(Y)$. That is, the rate $r$ controls the trade-off, between the excessive capital holding and the expected shortfall of loss, for the optimal capital. Such balancing acts with competing goals are widely known in practice for capital setting. However, discussions of balancing effect were largely restricted to risk measures, not in the context of risk aggregation and capital allocation.

Finally, in the conventional two-step procedure, it is unclear how diversification benefit contributes to the reduction for each $\mathrm{LOB}$ /risk from its standalone capital to allocated capital. The allocation of diversified capital is often done by ad-hoc methods. Take for example a common approach in the industry to allocate economic capital of a banking group to various business units [see (Resti and Sironi 2007, Chapter 23)]. Denote the standalone economic capital for the $i$ th $\mathrm{LOB} /$ risk by $E C_{i}$ and the total economic capital by $E C$. Then it is often the case that

$$
\sum_{i=1}^{n} E C_{i}>E C .
$$

The diversified economic capital for each LOB/risk, which is the actual amount redistributed, can be determined by some proportional apportionment method

$$
D E C_{i}=\frac{E C_{i}}{\sum_{j=1}^{n} E C_{j}} E C .
$$

While such an apportionment method is easy to use, it lacks meaningful justification. The method does not take in account the actual contribution made by each LOB/risk to the corporate overall risk profile. Figure 3 further illustrates these.

The novelty of this paper is to introduce a holistic approach in a quantitative framework, which addresses conflicting priorities between the capital management and the risk management, as well as the competing interests among the individual parts and the whole corporate, all in one single step to achieve Pareto optimality. By default, the holistic principle is free of the potential incompatibility of model assumptions in risk aggregation and capital allocation, and takes the capital management perspective into consideration. Most notably, the holistic principle provides a natural structural relationship among standalone capitals, aggregate capital, allocated capitals, and diversification benefit, which is consistent with the current market practice of allocating diversified capital, as illustrated above. In addition, the new framework 
enables users to consider a large family of risk measures for capital setting, such as the VaR, TVaR, and Conditional Tail Expectation (CTE), etc.

This paper also reviews some flaws of the widely adopted variance-covariance approach for risk aggregation and Euler allocation principle for capital allocation. The variance-covariance approach only captures diversification benefit through linear dependence. When losses are non-elliptically distributed, the variance-covariance approach does not necessarily provide a sufficient aggregate capital protection for the corporate or its units. While the Euler allocation principle is a straightforward and scientifically sound approach to allocation, it does not have a clear interpretation of diversified capital. Moreover, even if the losses are multivariate normally distributed, the Euler allocation principle could lead to severe under-reserving or over-reserving, when the losses are almost perfectly negatively correlated. As we shall demonstrate by numerical examples in Sect. 3.3 below, all such issues above can be resolved by the holistic approach.

The rest of the paper is organized as follows. Section 2 provides a bird's-eye view of competing components for aggregation and allocation as well as the technical definition of the holistic principle for risk aggregation and capital allocation. Examples of holistic principles with no constraint on the range of capitals are provided in Sect. 3. More sophisticated holistic principles with constraints can be found in Sect. 4. All holistic principles introduced in Sects. 3 and 4 are consistent with and natural extensions of the practice in the banking and insurance industries as shown in Fig. 3. We conclude the paper with parting remarks in Sect. 5. All proofs, if provided, are relegated to appendices.

\section{Holistic principle}

In the two-level corporate hierarchy, we take into account the interests of all individual LOBs/risks at the bottom level and that of the corporate as the top level. There are two competing management goals for each entity, namely, capital management and risk management, that affect how capital is aggregated and allocated. It is critical to first understand what is the need of each entity within the corporate via its optimal capital.

\subsection{Individual risk or line of business}

\subsubsection{Capital management}

For each individual LOB/risk $i=1,2, \ldots, n$, there could be different types of capital management objectives.

- [Minimal cost of capital $]$ Capitals are expensive as they must be held in the form of liquid assets, which usually have lower expected returns than other assets. If the opportunity cost of holding capital is proportion of the size of capital, then the optimal capital should be set at the lowest possible value, i.e. $K_{i}^{C}=\min K_{i}$.

- [Target capital $]$ It is unusual for big firms to rewrite their capital requirements substantially over a short period of time, as large fluctuations in accounting books raise red flags for auditors and regulators. If the capital level $c_{i}$ is known as the historical norm of allocated capital to this particular LOB/risk, then large deviation from such a norm should be discouraged. Thus, the optimal capital should be $K_{i}^{C}=c_{i}$.

One of the rationale for these choices of capital level, either the minimal or target capital, can be motivated by minimizing an objective function $C_{i}$, as a function of the allocated capital 
Table 1 Optimal capitals for capital management

\begin{tabular}{lll}
\hline & $C_{i}\left(K_{i}\right)$ & $K_{i}^{C}$ \\
\hline Target capital & $\left(c_{i}-K_{i}\right)^{2}$ & $c_{i}$ \\
Minimal cost of capital & $K_{i}$ & $\min K_{i}$ \\
\hline
\end{tabular}

Table 2 Optimal capitals for risk management

\begin{tabular}{lll}
\hline & $h_{i}\left(X_{i}\right)$ & $K_{i}^{R}=\mathbb{E}\left[X_{i} h_{i}\left(X_{i}\right)\right]$ \\
\hline Distortion risk measure & $D_{g_{i}}$ & $\rho_{g_{i}}\left(X_{i}\right)$ \\
Conditional expectation & $\frac{\mathbb{1}_{\left\{X_{i}>x_{i}\right\}}}{\mathbb{P}\left(X_{i}>x_{i}\right)}$ & $\mathbb{E}\left[X_{i} \mid X_{i}>x_{i}\right]$ \\
Entropic risk measure & $\int_{0}^{1} \frac{e^{t \gamma_{i} X_{i}}}{\mathbb{E}\left[e^{t \gamma_{i} X_{i}}\right]} d t$ & $\frac{1}{\gamma_{i}} \ln \left(\mathbb{E}\left[e^{\gamma_{i} X_{i}}\right]\right)$ \\
Standard deviation principle & $1+a_{i} \frac{X_{i}-\mathbb{E}\left[X_{i}\right]}{\sigma\left(X_{i}\right)}$ & $\mathbb{E}\left[X_{i}\right]+a_{i} \sigma\left(X_{i}\right)$ \\
Esscher principle & $\frac{e^{b_{i} X_{i}}}{\mathbb{E}\left[e^{b_{i} X_{i}}\right]}$ & $\frac{\mathbb{E}\left[X_{i} e^{b_{i} X_{i}}\right]}{\mathbb{E}\left[e^{b_{i} X_{i}}\right]}$ \\
\hline
\end{tabular}

$K_{i}$; if the objective $C_{i}\left(K_{i}\right)=K_{i}$, the optimal capital $K_{i}^{C}=\min K_{i}$, i.e. minimal cost of capital; if the objective $C_{i}\left(K_{i}\right)=\left(c_{i}-K_{i}\right)^{2}$, the optimal capital for capital management $K_{i}^{C}=c_{i}$, i.e. target capital. Table 1 summarizes these two optimal capitals for each individual capital management objective.

\subsubsection{Risk management}

It is fairly common in practice that, for each individual LOB/risk $i=1,2, \ldots, n$, the optimal capital for risk management $K_{i}^{R}$ is determined for the loss $X_{i}$ by $\rho_{i}\left(X_{i}\right)$, for some risk measure $\rho_{i}$ such as the VaR, TVaR, and CTE. It should be pointed out that these risk measures are in fact results of least squares optimization of risk residuals.

Each entity's risk management goal is to minimize an objective $R_{i}$, as a function of both the allocated capital $K_{i}$ and the loss $X_{i}$, which is given by $R_{i}\left(K_{i} ; X_{i}\right)$. While $R_{i}$ may take other forms, we shall only consider the particular case of mean squared risk residual, i.e. $R_{i}\left(K_{i} ; X_{i}\right)=\mathbb{E}\left[\left(X_{i}-K_{i}\right)^{2} h_{i}\left(X_{i}\right)\right]$, where $h_{i}\left(X_{i}\right)$ is a weighted penalty function for which $\mathbb{E}\left[h_{i}\left(X_{i}\right)\right]=1$. Such a risk residual objective with the weighted penalty function originated in Heilmann (1989), which was further explored in Laeven and Goovaerts (2004), Furman and Zitikis (2008a) and Furman and Zitikis (2008b). It measures the deviation of actual loss from the allocated capital intended to cover the loss. The square function is used to penalize large capital shortfall or overload. As a prudent risk management practice aims to control the risk residual, the optimal capital to meet the individual risk management objective is given by

$$
K_{i}^{R}=\arg \min R_{i}\left(K_{i} ; X_{i}\right)=\mathbb{E}\left[X_{i} h_{i}\left(X_{i}\right)\right] .
$$

Due to the flexibility of the weighted penalty function $h_{i}$, such an optimal capital includes a large family of risk measures $\rho_{i}$, as shown in Table 2, which is outlined in Furman and Zitikis (2008b) and Dhaene et al. (2012). 
Some technical conditions are necessary for the special cases in Table 2. For distortion risk measure, $g_{i}:[0,1] \rightarrow[0,1]$ is the non-decreasing distortion function with $g_{i}(0)=0$ and $g_{i}(1)=1$, and $D_{g_{i}}$ is denoted as the Radon-Nikodym derivative of the probability measure $\mathbb{Q}_{i}$, defined by $\mathbb{Q}_{i}(E)=\left(g_{i} \circ \mathbb{P}\right)(E)$, for any $E \in \mathcal{F}$, with respect to the physical probability measure $\mathbb{P}$. For conditional expectation, the real-valued constant $x_{i}$ is independent of the allocated capital $K_{i}$; if $x_{i}$ is given by the VaR of the loss $X_{i}$, it reduces to the CTE. For entropic risk measure, the positive constant $\gamma_{i}$ is the risk aversion parameter. For standard deviation principle, the constant $a_{i}$ is non-negative. For Esscher principle, the constant $b_{i}$ is positive.

Note that the risk management objective is no different from the mean squared error in statistics literature. It can be rewritten as $\mathbb{E}^{\mathbb{Q}_{i}\left(X_{i}\right)}\left[\left(X_{i}-K_{i}\right)^{2}\right]$ with a loss-dependent probability measure $\mathbb{Q}_{i}$. Therefore, the optimal capital, $K_{i}^{R}=\mathbb{E}\left[X_{i} h_{i}\left(X_{i}\right)\right]$, shall be referred as the least squares capital in this paper for brevity. Herein, the capital shortfall or overload is penalized with equal weights. This has recently been generalized with unequal weights for the capital overload and shortfall in Chen et al. (2020) which introduces the weighted least squares capital.

\subsection{Corporate-capital and risk managements}

Similarly, at the corporate level, on one hand, the optimal capital $K^{C}$ for capital management purpose is set by minimizing an objective $C(K)$ in line with Table 1 . On the other hand, the optimal capital $K^{R}$ for risk management purpose is determined by a risk measure $\rho(S)$, which minimizes the objective $R(K ; S)=\mathbb{E}\left[(S-K)^{2} h(S)\right]$, for some weighted penalty function $h(S)$ for which $\mathbb{E}[h(S)]=1$; appropriate choices of the weighted penalty functions herein follow similarly as in Table 2 .

\subsection{Pareto optimality}

Taking capital and risk managements for all individual LOBs/risks and the corporate level into considerations, Fig. 4 shows the competing interests and conflicting priorities in the capital allocation practice. The holistic principle for risk aggregation and capital allocation proposes to strike a balance among these interests and priorities via Pareto optimality.

Pareto optimality is a well-known economic concept in any multi-objective optimization problem; this optimality leads to an efficient capital allocation such that it is impossible to improve any allocation objective in the corporate, without comprising any other allocation objectives. Even though every component is allocated a sub-optimal capital, in the sense that it is not given by its optimal capital, the entire corporate achieves its best possible equilibrium in the sense of Pareto optimality.

Definition 1 The holistic principle for risk aggregation and capital allocation maps the random vector $\left(X_{1}, X_{2}, \ldots, X_{n}\right)$ to a constant vector $\left(K_{1}^{H}, K_{2}^{H}, \ldots, K_{n}^{H}\right)$, which is given by a minimizer of the following optimization problem:

$$
\inf _{\left(K_{1}, K_{2}, \ldots, K_{n}\right) \in \mathcal{K}} \sum_{i=1}^{n} v_{i} C_{i}\left(K_{i}\right)+\sum_{i=1}^{n} \omega_{i} R_{i}\left(K_{i} ; X_{i}\right)+v C(K)+\omega R(K ; S),
$$

where $\mathcal{K} \subseteq \mathbb{R}^{n}$ is a non-empty set of admissible capital allocations; $v_{i}, \omega_{i}$, for $i=1,2, \ldots, n$, $\nu$, and $\omega$ are non-negative deterministic weights, with at least one of them being positive. Recall that $K=K_{1}+K_{2}+\cdots+K_{n}$ is the aggregate capital. 


\begin{tabular}{|c|c|c|}
\hline & Capital management & Risk management \\
\hline 1-st LOB/Risk & $\inf C_{1}\left(K_{1}\right)$ & $\inf R_{1}\left(K_{1} ; X_{1}\right)$ \\
\hline 2-nd LOB/Risk & $\inf C_{2}\left(K_{2}\right)$ & $\inf R_{2}\left(K_{2} ; X_{2}\right)$ \\
\hline$\vdots$ & $\vdots$ & : \\
\hline$n$-th LOB/Risk & $\inf C_{n}\left(K_{n}\right)$ & $\inf R_{n}\left(K_{n} ; X_{n}\right)$ \\
\hline Corporate & $\inf C(K)$ & $\inf R(K ; S)$ \\
\hline
\end{tabular}

Fig. 4 Multi-objective capital allocation-competing interests and conflicting priorities

The non-negative deterministic Pareto optimality weights, $v_{i}, \omega_{i}$, for $i=1,2, \ldots, n$, $\nu$, and $\omega$, signify the relative bargaining power of each component in the capital allocation practice. The larger the weight is, the stronger influence the corresponding allocation objective has on the holistic principle for risk aggregation and capital allocation.

The non-empty admissible capital allocation set $\mathcal{K}$ contains all possible capital allocations. Examples include, but are not limited to,

- [Unconstrained case $] \mathcal{K}=\mathbb{R}^{n}$. This case is to be addressed in Sect. 3 .

- $[$ Constrained case-bounded capitals $] \mathcal{K}=\left[\underline{b}_{1}, \bar{b}_{1}\right] \times\left[\underline{b}_{2}, \bar{b}_{2}\right] \times \cdots \times\left[\underline{b}_{n}, \bar{b}_{n}\right]$. This case is to be investigated in Sect. 4.

- [Constrained case-fixed budget $] \mathcal{K}=\left\{K_{1}+\cdots+K_{n}=C\right\}$ for some fixed aggregate capital $C$. This case reduces to the classical problem of capital allocation, which is already well studied in Dhaene et al. (2012).

Before closing this section, we compare our proposed holistic principle in (1) with some other approaches in the literature.

Firstly, the diversification effect by the holistic principle is fundamentally different from that in many other existing risk aggregation methods. For example, the diversification by the full base correlation matrix approach developed in Filipović (2009) results from the assumption of risk correlations. The holistic approach produces the diversification through a balancing act of competing objectives.

Secondly, the holistic principle extends existing methods for capital allocation pioneered by the work of Dhaene et al. (2012). In particular, the proposed holistic principle in (1) is inspired by (31) in Dhaene et al. (2012). There are also four major differences between the capital allocation in Dhaene et al. (2012) and the holistic principle in this paper. (i) The holistic principle extends beyond risk management objectives in the capital allocation principle in Dhaene et al. (2012) and takes into account capital management objectives. (ii) The weights in the holistic principle are natural from a game theoretical perspective, in particular the Pareto optimality for competing interests and conflicting priorities, while the weights in Dhaene et al. (2012) are motivated by business exposures. (iii) The holistic principle considers the dependence structure of LOBs/risks via the corporate risk management objective instead of the weighted penalty function. (iv) Dhaene et al. (2012) is in essence 
the holistic principle with a fixed aggregate capital, which reduces to a capital allocation problem.

Thirdly, the holistic principle complements the research on systemic risk measure by extending the notion of systemic risk measure to Pareto optimality. Systemic risk measure in the literature can be cast as a result from an optimization problem in the form of (1) without risk management objectives and with $\mathcal{K}$ being an acceptance set. The unified framework of systemic risk measure was introduced and studied extensively by Biagini et al. (2019). In their work, the optimized objective function is considered the systemic risk measure. In contrast, the holistic principle uses optimizers (solutions to the optimization of objective function) as allocated capitals.

Lastly, the holistic principle provides an alternative to the unification of top-down and bottom-up capital allocation methods proposed by Furman et al. (2020). In their work, the top-down approach refers to proportional allocation rules of total capital determined by some risk measure of aggregate risk and the bottom-up approach means allocation rules that assign a capital to each individual risk based on the joint distribution of its loss and the aggregate risk. The concept is developed by a series of work by the authors on weighted allocation rules. As we shall remark after Theorem 1, the holistic principle builds upon weighted allocations, but takes a game theoretical approach, in particular the Pareto optimality, on competing interests and conflicting priorities for standalone optimal capitals.

\section{Unconstrained case}

\subsection{Targeted and least squares capital allocation}

Consider first the case where the corporate and individual LOBs/risks all have a priori set of capital targets, $c, c_{1}, c_{2}, \ldots, c_{n}$. While all entities aim to limit deviation from their targets, they also want to minimize risk residuals for better risk management.

Problem 1 The unconstrained targeted and least squares holistic principle $\left(K_{1}^{H}, K_{2}^{H}, \ldots\right.$, $\left.K_{n}^{H}\right)$ solves

$\inf _{\left(K_{1}, K_{2}, \ldots, K_{n}\right) \in \mathbb{R}^{n}} \sum_{i=1}^{n} v_{i}\left(c_{i}-K_{i}\right)^{2}+\sum_{i=1}^{n} \omega_{i} \mathbb{E}\left[\left(X_{i}-K_{i}\right)^{2} h_{i}\left(X_{i}\right)\right]+v(c-K)^{2}+\omega \mathbb{E}\left[(S-K)^{2} h(S)\right]$.

The following theorem states the closed-form expressions for the unconstrained targeted and least squares holistic aggregate and allocated capitals.

Theorem 1 The unconstrained targeted and least squares holistic aggregate and allocated capitals are given by

$$
\begin{aligned}
K^{H} & =\bar{K}+A\left(\sum_{r=1}^{n} \bar{K}_{r}-\bar{K}\right), \\
K_{i}^{H} & =\bar{K}_{i}-B_{i}\left(\sum_{r=1}^{n} \bar{K}_{r}-\bar{K}\right),
\end{aligned}
$$

for any $i=1,2, \ldots, n$, where

$$
\bar{K}_{i}=\alpha_{i} K_{i}^{C}+\beta_{i} K_{i}^{R}, \quad \bar{K}=\alpha K^{C}+\beta K^{R},
$$




$$
\begin{aligned}
K_{i}^{C} & =c_{i}, \quad K_{i}^{R}=\rho_{i}\left(X_{i}\right)=\mathbb{E}\left[X_{i} h_{i}\left(X_{i}\right)\right], \quad K^{C}=c, \quad K^{R}=\rho(S)=\mathbb{E}[\operatorname{Sh}(S)], \\
A & =\frac{\frac{1}{v+\omega}}{\frac{1}{v+\omega}+\sum_{r=1}^{n} \frac{1}{v_{r}+\omega_{r}}}, \quad B_{i}=\frac{\frac{1}{v_{i}+\omega_{i}}}{\frac{1}{v+\omega}+\sum_{r=1}^{n} \frac{1}{v_{r}+\omega_{r}}}, \\
\alpha_{i} & =\frac{v_{i}}{v_{i}+\omega_{i}}, \quad \beta_{i}=\frac{\omega_{i}}{v_{i}+\omega_{i}}, \quad \alpha=\frac{v}{v+\omega}, \quad \beta=\frac{\omega}{v+\omega} .
\end{aligned}
$$

Owing to the analogy between the optimization problem (1) for the holistic principle and that for the capital allocation principle in (31) of Dhaene et al. (2012), we obtain holistic allocated capitals in a similar form (3) as those optimal allocated capitals in equation (34) of Dhaene et al. (2012). One should note that Dhaene et al. (2012) considered a more general weighted penalty function. In particular, that could be depending on a market portfolio $\zeta_{M}$. While the holistic principle herein does not take the market driven term into account, it can be easily extended by incorporating a similar objective function as follows.

$$
\begin{aligned}
\inf _{\left(K_{1}, K_{2}, \ldots, K_{n}\right) \in \mathbb{R}^{n}} & \sum_{i=1}^{n} v_{i}\left(c_{i}-K_{i}\right)^{2}+\sum_{i=1}^{n} \omega_{i} \mathbb{E}\left[\left(X_{i}-K_{i}\right)^{2} h_{i}\left(X_{i}\right)\right]+\sum_{i=1}^{n} \omega_{i}^{M} \mathbb{E}\left[\left(X_{i}-K_{i}\right)^{2} \zeta_{M}\right] \\
& +v(c-K)^{2}+\omega \mathbb{E}\left[(S-K)^{2} h(S)\right]+\omega^{M} \mathbb{E}\left[(S-K)^{2} \zeta_{M}\right] .
\end{aligned}
$$

It is straightforward to show that the holistic aggregate and allocated capitals in Theorem 1 are generalized accordingly, together with $\rho^{M}\left(X_{i}\right)=\mathbb{E}\left[X_{i} \zeta_{M}\right]$ and $\rho^{M}(S)=\mathbb{E}\left[S \zeta_{M}\right]$. It is also possible to recover weighted allocation rules developed in the literature by replacing $h_{i}\left(X_{i}\right)$ by $h_{i}(S)$. For brevity, we do not consider these more general terms in the remaining of this paper.

\subsubsection{Economic interpretations}

The above expressions for the targeted and least squares holistic capital are composed of three crucial components, namely, the optimal aggregate capital $\bar{K}$ in the corporate level, the optimal standalone capital $\bar{K}_{i}$ for each individual LOB/risk, and the potential diversification benefit. The way they are used to determine holistic capitals can be summarized in Fig. 5.

- [Optimal aggregate capital at the corporate level by horizontal balancing] This corresponds to the term $\alpha K^{C}+\beta K^{R}$, which is the optimal aggregate capital when only the corporate level is considered, with $v_{i}=\omega_{i}=0$, for all $i=1,2, \ldots, n$ :

$$
\bar{K}=\arg \min \{v C(K)+\omega R(K ; S)\}=\alpha K^{C}+\beta K^{R} .
$$

Since $\alpha+\beta=1$, such an optimal aggregate capital carries an arithmetic weighting for the two optimal capitals $K^{C}$ and $K^{R}$ for capital management and risk management purposes. This can be viewed as a balancing act of competing interests horizontally in Fig. 4, which depicts the trade-offs between the two management objectives in the corporate level. The objective with a higher weight would exert a stronger influence on the resulting optimal aggregate capital.

- [Optimal standalone capital for each LOB/risk by horizontal balancing] This corresponds to the term $\alpha_{i} K_{i}^{C}+\beta_{i} K_{i}^{R}$, which is the optimal standalone capital when only the $i$ th LOB/risk is considered, for any $i=1,2, \ldots, n$, with $v_{j}=\omega_{j}=0$, for all $j=1,2, \ldots, n$ that $j \neq i$, and $v=\omega=0$ :

$$
\bar{K}_{i}=\arg \min \left\{v_{i} C_{i}\left(K_{i}\right)+\omega_{i} R_{i}\left(K_{i} ; X_{i}\right)\right\}=\alpha_{i} K_{i}^{C}+\beta_{i} K_{i}^{R} .
$$




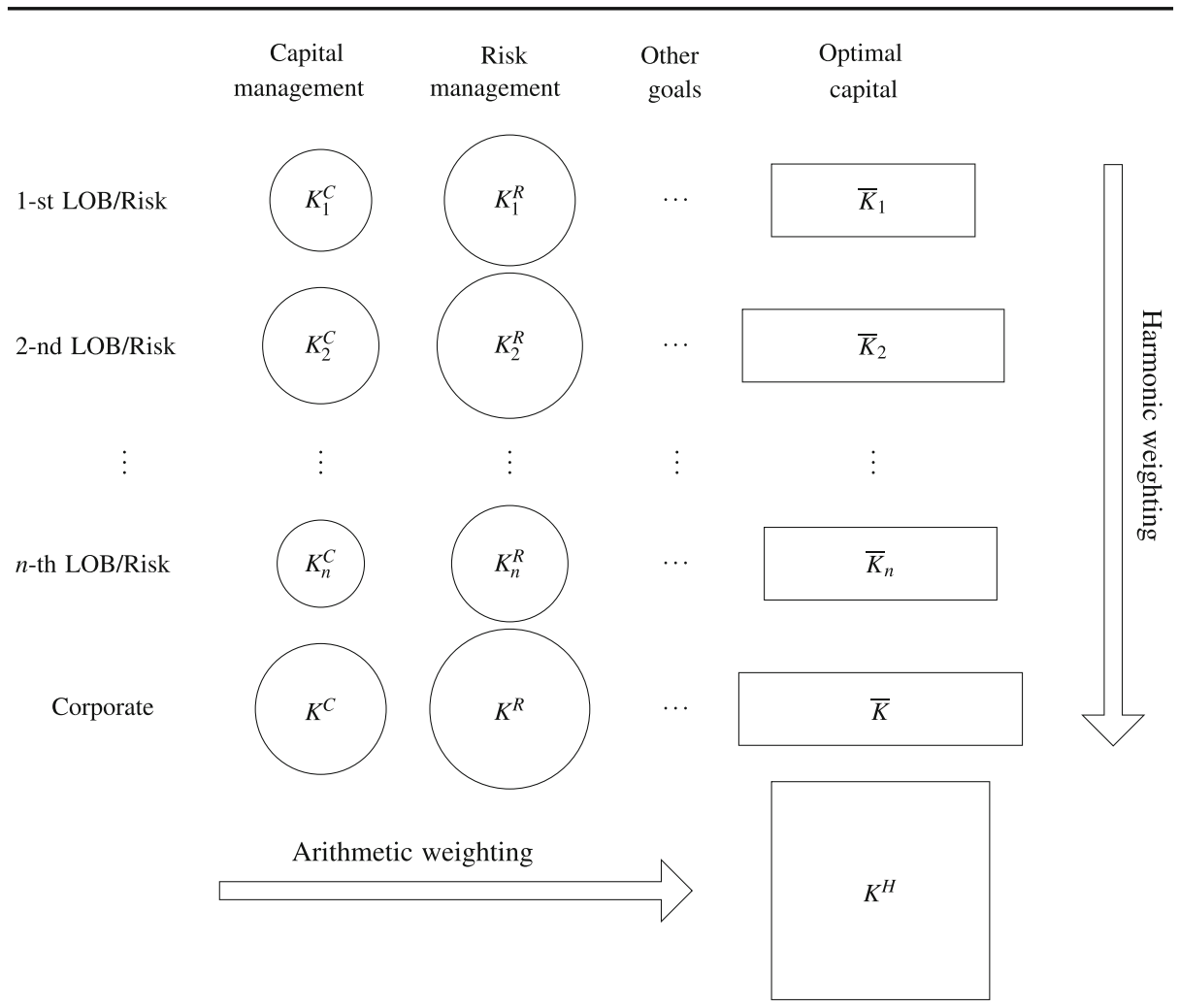

Fig. 5 Holistic aggregate and allocated capitals_-balancing of optimal capitals

Similarly, since $\alpha_{i}+\beta_{i}=1$, the optimal standalone capital for the $i$ th loss $X_{i}$ is the arithmetic average of the two optimal capitals $K_{i}^{C}$ and $K_{i}^{R}$ for capital management and risk management. This can be perceived as a balancing act of competing interests horizontally in Fig. 4, which depicts the trade-offs between the two management objectives at the $i$ th individual level of LOB/risk. The objective with a higher weight would exert more influence on the resulting optimal standalone capital.

- [Diversification benefit $]$ This corresponds to the difference term, between the aggregation of optimal standalone capitals for all LOBs/risks, and the optimal aggregate capital for the corporate:

$$
\sum_{r=1}^{n} \bar{K}_{r}-\bar{K} .
$$

If the difference is positive, there is a diversification benefit among the units within the corporate. This amount indicates risk reduction at the corporate level for its diversified portfolio. In theory, the difference could also be negative, which indicates exacerbated risk due to a concentration effect. For the ease of exposition, we shall only use the interpretation for all formulas in this section with the diversification benefit. The opposite can be said for the concentration effect.

In the current industry practice, the diversified capital is often distributed to each LOB/risk in proportion to its standalone capital, as shown in Fig. 3. Such a method is heuristic and 


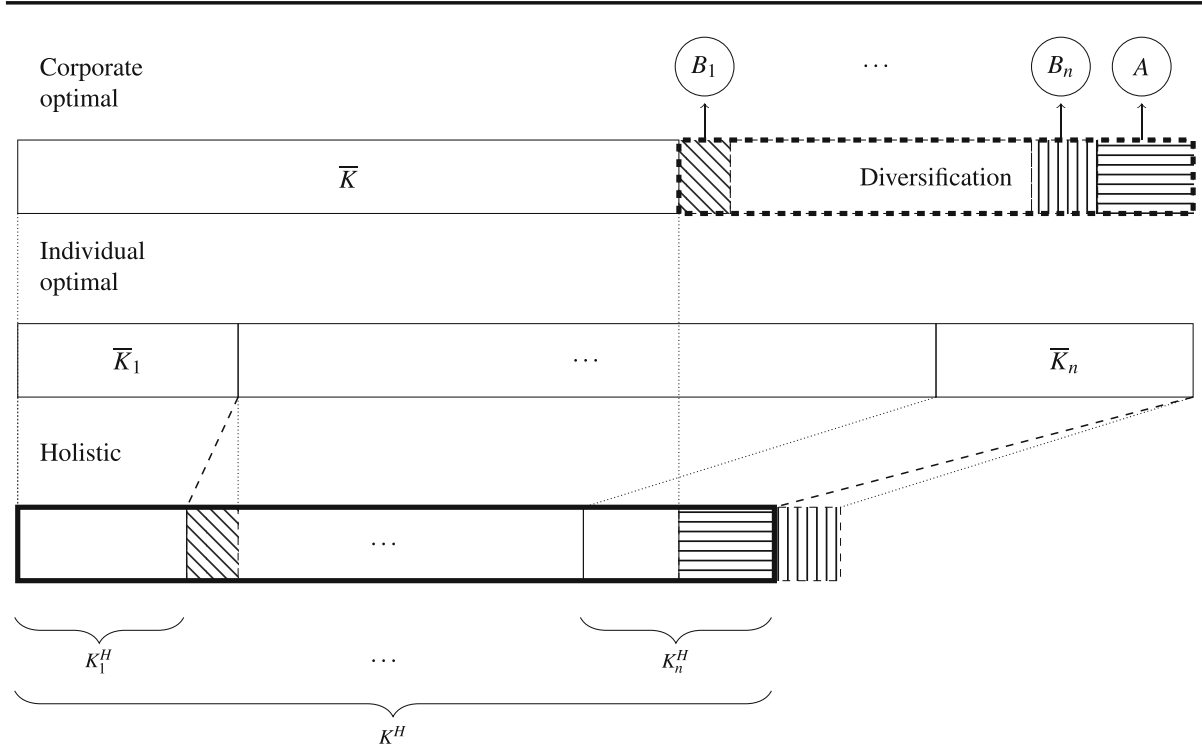

Fig. 6 Holistic aggregate and allocated capitals—relationships with optimal aggregate and standalone capitals

lacks meaningful justification. The holistic principle for risk aggregation and capital allocation demonstrates a surprising similarity to the industry standard but is based on scientific reasoning. The connection of the holistic aggregate and allocated capitals, in relation to optimal standalone and aggregate capitals for all LOBs/risks and in the corporate level, is best illustrated in Figs. 5 and 6 .

- [Decomposition of reduction by diversification benefit] The targeted and least squares holistic allocated capital is clearly a compromise made between optimal standalone capital for each LOB/risk and the optimal aggregate capital at the corporate level. The capital allocation formula (3) shows that each $\mathrm{LOB}$ /risk gives up some of its optimal standalone capital determined by a percentage $B_{i}$ of the reduction by diversification. It is remarkable that the decomposition of reduced capital by diversification is based on harmonic weighting, since

$$
A=\frac{\frac{1}{v+\omega}}{\frac{1}{v+\omega}+\sum_{r=1}^{n} \frac{1}{v_{r}+\omega_{r}}}, \quad B_{i}=\frac{\frac{1}{v_{i}+\omega_{i}}}{\frac{1}{v+\omega}+\sum_{r=1}^{n} \frac{1}{v_{r}+\omega_{r}}}, \quad A+\sum_{i=1}^{n} B_{i}=1 .
$$

When a particular LOB/risk is viewed as significantly more important, i.e. the weight $v_{i}+\omega_{i}$ is comparatively larger, the weight $B_{i}$ is smaller, and hence the LOB/risk is required to cut less from its optimal standalone capital.

- [Vertical balancing] In view of its competing interests with LOBs/risks, the corporate holds more capital than its optimal aggregate capital, and the increased amount is also based on the harmonic weighting, i.e.

$$
K^{H}=(1-A) \bar{K}+A \sum_{i=1}^{n} \bar{K}_{i} .
$$

When the corporate has a higher priority for its optimality, i.e. the weight $v+\omega$ is larger, the weight $A$ is smaller, and hence brings the holistic aggregate capital $K^{H}$ closer to its 
optimal aggregate capital $\bar{K}$. The harmonic weighting is a result of the vertical balancing in Fig. 4.

\subsubsection{Sensitivity with respect to relative bargaining powers}

The targeted and least squares holistic aggregate and allocated capitals in (2) and (3) clearly depend on the relative bargaining powers of all components in the corporate hierarchy, either for capital or risk managements, and at the individual LOB/risk, or the corporate levels. The following proposition summarizes the marginal sensitivity of the holistic capital, with respect to each relative weight of individual LOB/risk, and the corporate.

Proposition 1 (a) Suppose that the diversification benefit exists, i.e.,

$$
\bar{K} \leq \sum_{r=1}^{n} \bar{K}_{r} .
$$

For any $i=1,2, \ldots, n$, if $v_{i}+\omega_{i}$ increases, without changing $\alpha_{i}$ and $\beta_{i}$, then both $K_{i}^{H}$ and $K^{H}$ increase, while $K_{j}^{H}$, for all $j=1,2, \ldots, n$ that $j \neq i$, decrease; if $v+\omega$ increases, without changing $\alpha$ and $\beta$, all $K_{i}^{H}$, for $i=1,2, \ldots, n$, and $K^{H}$ decrease.

(b) Suppose that the concentration effect exists, i.e.,

$$
\bar{K} \geq \sum_{r=1}^{n} \bar{K}_{r} .
$$

For any $i=1,2, \ldots, n$, if $v_{i}+\omega_{i}$ increases, without changing $\alpha_{i}$ and $\beta_{i}$, then both $K_{i}^{H}$ and $K^{H}$ decrease, while $K_{j}^{H}$, for all $j=1,2, \ldots, n$ that $j \neq i$, increase; if $v+\omega$ increases, without changing $\alpha$ and $\beta$, all $K_{i}^{H}$, for $i=1,2, \ldots, n$, and $K^{H}$ increase.

These sensitivity results can be interpreted with the aid of Fig. 6. Since $v_{i}+\omega_{i}$ or $v+\omega$ are increased with keeping $\alpha_{i}, \beta_{i}, \alpha$, or $\beta$ as constants, neither the optimal aggregate capital at the corporate level, nor the optimal standalone capital for each individual LOB/risk, is affected. The lengths of the corporate bar and the total of individual LOB/risk bar remain unchanged in Fig. 6. All moving parts lie in the diversification benefit.

In any case, the holistic aggregate capital $K^{H}\left(=K_{1}^{H}+K_{2}^{H}+\cdots+K_{n}^{H}\right)$ can be thought of as the mid-point of a rope in a tug-of-war; the optimal aggregate capital in the corporate level is on one end of the rope, while all optimal standalone capitals for individual LOBs/risks are on the other end. For brevity, only the results in part (a) will be discussed, since, again, opposite interpretations hold for the concentration effect. In this case, the optimal aggregate capital in the corporate level is less than the holistic aggregate capital $K^{H}$. Therefore, when the corporate increases its relative bargaining power, the holistic aggregate capital $K^{H}$ leans towards its optimal aggregate capital. Keep in mind that $A$ is the proportion of diversification benefit by which $K^{H}$ exceeds $\bar{K}$. Hence the corresponding component $A$ in the diversification benefit shrinks. Because the total diversification benefit is fixed, all other components $B_{1}, B_{2}, \ldots, B_{n}$ in the diversification benefit have to increase, and hence all holistic allocated capitals $K_{1}^{H}, K_{2}^{H}, \ldots, K_{n}^{H}$ are cut more from their corresponding optimal standalone capitals, and thus are reduced. Figure 7 demonstrates these changes.

Similar interpretations apply to the sensitivity analysis with respect to the relative bargaining power of the $i$ th individual LOB/risk. When the holistic allocated capital $K_{i}^{H}$ leans towards its optimal standalone capital, $K_{i}^{H}$ increases, which means that the corresponding 
Corporate

optimal

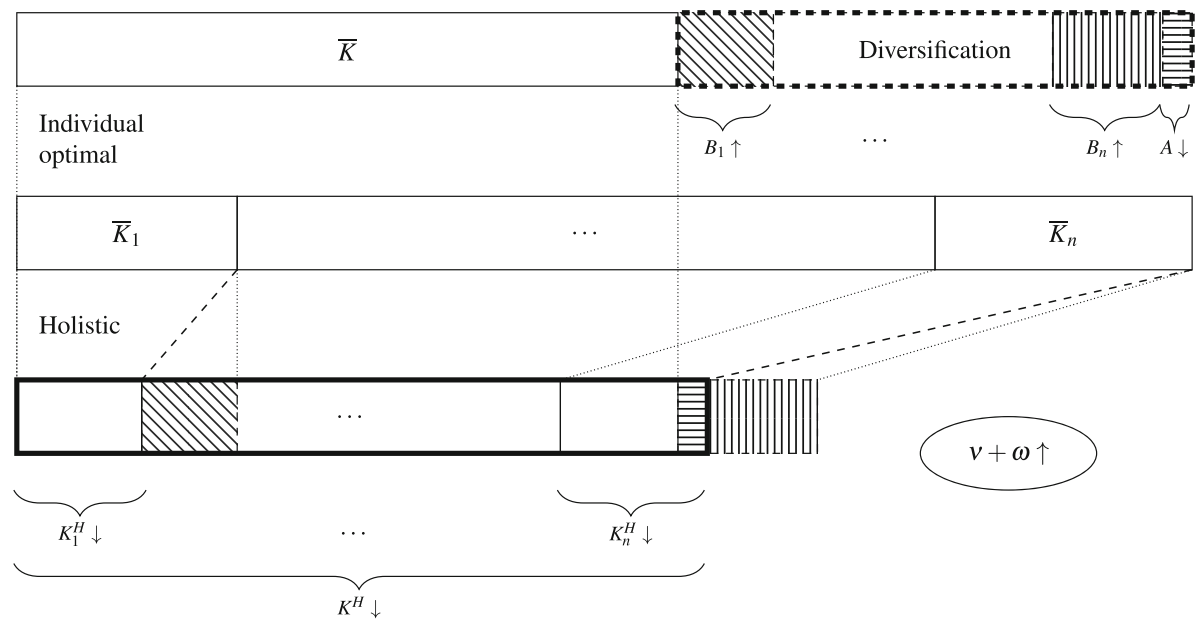

Fig. 7 Sensitivity with respect to bargaining power of corporate

component $B_{i}$ in the diversification benefit decreases. As the diversification amount is fixed, all other components $A$ and $B_{j}$ in the diversification benefit, for $j=1,2, \ldots, n$ that $j \neq i$, have to increase. Hence, the holistic allocated capitals $K_{j}^{H}$, for all $j=1,2, \ldots, n$ that $j \neq i$, decrease, as more are cut from their corresponding optimal standalone capitals. In this case, the holistic aggregate capital $K^{H}$ increases because more capital is added to the corresponding optimal aggregate capital.

The following proposition summarizes the marginal sensitivity of the holistic aggregate and allocated capitals, with respect to each relative bargaining power of capital or risk managements, and at the individual LOB/risk, or the corporate levels. Similar interpretations hold for these sensitivity results with the aid of Fig. 6, and hence are omitted.

Proposition 2 (a) For any $i=1,2, \ldots, n$, and for any $j=1,2, \ldots, n$ that $j \neq i$, when $K_{i}^{H} \leq K_{i}^{C}$, both $K_{i}^{H}$ and $K^{H}$ increase, while $K_{j}^{H}$ decreases, with $v_{i}$; when $K_{i}^{H} \geq K_{i}^{C}$, both $K_{i}^{H}$ and $K^{H}$ decrease, while $K_{j}^{H}$ increases, with $v_{i}$.

(b) For any $i=1,2, \ldots, n$, and for any $j=1,2, \ldots, n$ that $j \neq i$, when $K_{i}^{H} \leq K_{i}^{R}$, both $K_{i}^{H}$ and $K^{H}$ increase, while $K_{j}^{H}$ decreases, with $\omega_{i}$; when $K_{i}^{H} \geq K_{i}^{R}$, both $K_{i}^{H}$ and $K^{H}$ decrease, while $K_{j}^{H}$ increases, with $\omega_{i}$.

(c) When $K^{H} \leq K^{C}$, all $K_{i}^{H}$, for $i=1,2, \ldots, n$, and $K^{H}$ increase with $v$; when $K^{H} \geq K^{C}$, all $K_{i}^{H}$, for $i=1,2, \ldots, n$, and $K^{H}$ decrease with $v$.

(d) When $K^{H} \leq K^{R}$, all $K_{i}^{H}$, for $i=1,2, \ldots, n$, and $K^{H}$ increase with $\omega$; when $K^{H} \geq K^{R}$, all $K_{i}^{H}$, for $i=1,2, \ldots, n$, and $K^{H}$ decrease with $\omega$.

\subsubsection{Special cases: optimal capitals}

It is expected that, if a relative bargaining power of a particular component in the corporate hierarchy is sufficiently large, the targeted and least squares holistic aggregate and allocated capitals in (2) and (3) should be as close as possible to the optimal capital of that component. Indeed, the following corollary shows these convergence results. 
Corollary 1 For any $i=1,2, \ldots, n$,

$$
\lim _{\nu_{i} \rightarrow \infty} K_{i}^{H}=K_{i}^{C}, \quad \lim _{\omega_{i} \rightarrow \infty} K_{i}^{H}=K_{i}^{R}, \quad \lim _{\nu \rightarrow \infty} K^{H}=K^{C}, \quad \lim _{\omega \rightarrow \infty} K^{H}=K^{R} .
$$

\subsubsection{Stability with respect to allocation targets}

There are two additional inputs for targeted and least squares holistic aggregate and allocated capitals in (2) and (3), namely, risk measures $\rho_{1}, \rho_{2}, \ldots, \rho_{n}, \rho$, and capital allocation targets $c_{1}, c_{2}, \ldots, c_{n}, c$. As shown in Table 2 , different choices of the weighted penalty functions induce different risk measures. All economic interpretations and analysis apply regardless of particular choices for risk measures.

This paper has so far not imposed any rule for capital allocation targets. In practice, actual capitals are reported in accounting books and are expected not to fluctuate dramatically. Otherwise, it raises red flags for regulators and auditors. It makes sense to use holistic aggregate and allocated capitals from the previous period as capital targets for this period. Therefore, this section addresses the following question-does the holistic principle reach an equilibrium?

To this end, we consider a multi-period model where holistic aggregate and allocated capital from one period are treated as target capitals for the next period. Applying the targeted and least squares holistic principle for each period $t=1,2, \ldots$, we obtain holistic capital denoted by $K_{i}^{H}(t)$, for $i=1,2, \ldots, n$, and $K^{H}(t)$. By definition, these holistic capitals are dependent on their capital targets, which are denoted by $c_{i}(t)$, for $i=1,2, \ldots, n$, and $c(t)$. The following theorem states that, if capital targets are updated periodically by current holistic capitals, then the holistic principle converges to an equilibrium in the long-run.

Theorem 2 Suppose that the non-negative weights $\omega_{i}$, for $i=1,2, \ldots, n$, are positive, and risk measures $\rho_{1}, \rho_{2}, \ldots, \rho_{n}, \rho$ are time independent. If capital allocation targets are given by, for any $i=1,2, \ldots, n$ and $t=1,2, \ldots$,

$$
c_{i}(t)=K_{i}^{H}(t-1), \quad c(t)=K^{H}(t-1),
$$

then there exists an allocation $\left(K_{1}^{H}, K_{2}^{H}, \ldots, K_{n}^{H}\right) \in \mathbb{R}^{n}$ such that

$$
\lim _{t \rightarrow \infty} K_{1}^{H}(t)=K_{1}^{H}, \quad \lim _{t \rightarrow \infty} K_{2}^{H}(t)=K_{2}^{H}, \quad \ldots, \quad \lim _{t \rightarrow \infty} K_{n}^{H}(t)=K_{n}^{H},
$$

which is independent of initial capital targets $\left(K_{1}^{H}(0), K_{2}^{H}(0), \ldots, K_{n}^{H}(0)\right)$.

\subsection{Minimal and least squares capital allocation}

Here we consider another combination of capital management and risk management goals, which is to strike a balance between minimizing the cost of capital and risk residuals.

Problem 2 The unconstrained minimal and least squares holistic principle $\left(K_{1}^{H}, K_{2}^{H}, \ldots, K_{n}^{H}\right)$ solves

$$
\inf _{\left(K_{1}, K_{2}, \ldots, K_{n}\right) \in \mathbb{R}^{n}} \sum_{i=1}^{n} v_{i} K_{i}+\sum_{i=1}^{n} \omega_{i} \mathbb{E}\left[\left(X_{i}-K_{i}\right)^{2} h_{i}\left(X_{i}\right)\right]+v K+\omega \mathbb{E}\left[(S-K)^{2} h(S)\right] .
$$


Theorem 3 The unconstrained minimal and least squares holistic aggregate and allocated capitals in Problem 2 are given by

$$
\begin{aligned}
& K^{H^{\prime}}=\bar{K}^{\prime}+A^{\prime}\left(\sum_{r=1}^{n} \bar{K}_{r}^{\prime}-\bar{K}^{\prime}\right), \\
& K_{i}^{H^{\prime}}=\bar{K}_{i}^{\prime}-B_{i}^{\prime}\left(\sum_{r=1}^{n} \bar{K}_{r}^{\prime}-\bar{K}^{\prime}\right),
\end{aligned}
$$

for any $i=1,2, \ldots, n$, where

$$
\begin{aligned}
\bar{K}^{\prime} & =K^{R}-\frac{\delta}{2}, \quad \bar{K}_{i}^{\prime}=K_{i}^{R}-\frac{\delta_{i}}{2}, \\
K_{i}^{R} & =\rho_{i}\left(X_{i}\right)=\mathbb{E}\left[X_{i} h_{i}\left(X_{i}\right)\right], \quad K^{R}=\rho(S)=\mathbb{E}[S h(S)], \\
A^{\prime} & =\frac{\frac{1}{\omega}}{\frac{1}{\omega}+\sum_{r=1}^{n} \frac{1}{\omega_{r}}}, \quad B_{i}^{\prime}=\frac{\frac{1}{\omega_{i}}}{\frac{1}{\omega}+\sum_{r=1}^{n} \frac{1}{\omega_{r}}}, \quad \delta_{i}=\frac{v_{i}}{\omega_{i}}, \quad \delta=\frac{v}{\omega} .
\end{aligned}
$$

Note that the minimal and least squares holistic capitals, given by (4) and (5), share similar structures as the targeted and least squares holistic capitals, given by (2) and (3). Indeed, (4) and (5) also contain the three crucial components, namely,

- [Optimal aggregate capital at the corporate level] which is the optimal capital when only the corporate level is considered:

$$
\bar{K}^{\prime}=\arg \min \left\{v K+\omega \mathbb{E}\left[(S-K)^{2} h(S)\right]\right\}=K^{R}-\frac{\delta}{2} ;
$$

- [Optimal standalone capital for each $L O B /$ risk] which is the optimal capital when only the $i$ th $\mathrm{LOB} /$ risk is considered: for any $i=1,2, \ldots, n$,

$$
\bar{K}_{i}^{\prime}=\arg \min \left\{v_{i} K_{i}+\omega_{i} \mathbb{E}\left[\left(X_{i}-K_{i}\right)^{2} h_{i}\left(X_{i}\right)\right]\right\}=K_{i}^{R}-\frac{\delta_{i}}{2} ;
$$

- [Diversification benefit $] \sum_{r=1}^{n} \bar{K}_{i}^{\prime}-\bar{K}^{\prime}$, which could be positive or negative (concentration effect).

Moreover, the holistic capitals, given by (4) and (5), are again shown to be the adjustment of optimal aggregate and standalone capitals by portions of the diversification benefit. The allocation of reduction by diversification is also determined by harmonic weighting.

The following proposition summarizes the marginal sensitivity of the minimal and least squares holistic principle with respect to relative bargaining power of capital or risk managements, and at the individual LOB/risk, or the corporate levels.

Proposition 3 (a) For any $i=1,2, \ldots, n$, and for any $j=1,2, \ldots, n$ that $j \neq i$, both $K_{i}^{H^{\prime}}$ and $K^{H^{\prime}}$ decrease, while $K_{j}^{H^{\prime}}$ increases, with $v_{i}$.

(b) For any $i=1,2, \ldots, n$, and for any $j=1,2, \ldots, n$ that $j \neq i$, when $K_{i}^{H^{\prime}} \leq K_{i}^{R}$, both $K_{i}^{H^{\prime}}$ and $K^{H^{\prime}}$ increase, while $K_{j}^{H^{\prime}}$ decreases, with $\omega_{i}$; when $K_{i}^{H^{\prime}} \geq K_{i}^{R}$, both $K_{i}^{H^{\prime}}$ and $K^{H^{\prime}}$ decrease, while $K_{j}^{H^{\prime}}$ increases, with $\omega_{i}$.

(c) All $K_{i}^{H^{\prime}}$, for $i=1,2, \ldots, n$, and $K^{H^{\prime}}$ decrease with $v$.

(d) When $K^{H^{\prime}} \leq K^{R}$, all $K_{i}^{H^{\prime}}$, for $i=1,2, \ldots, n$, and $K^{H^{\prime}}$ increase with $\omega$; when $K^{H^{\prime}} \geq$ $K^{R}$, all $K_{i}^{H^{\prime}}$, for $i=1,2, \ldots, n$, and $K^{H^{\prime}}$ decrease with $\omega$. 
One can also interpret sensitivity results with the aid of similar graphs as in Figs. 6 and 7 . Note that the results in parts (b) and (d) of Proposition 3 resemble those in Proposition 2. However, since the relative bargaining powers of capital management herein affect only optimal aggregate and standalone capitals and the diversification benefit, but not the harmonic weighting, results in parts (a) and (c) of Proposition 3 are simplified without any sub-case conditions.

\subsection{Comparisons with classical methods}

This section devotes to comparing the least squares holistic principle, in (2) and (3), or in (4) and (5), with the classical variance-covariance approach and the Euler allocation principle. For fair comparisons, we do not consider capital management objectives, with $v_{1}=v_{2}=\cdots=v_{n}=v=0$, since the above-mentioned classical methods do not take capital management into account. Assume further that risk measures for all entities are the same, i.e. $\rho_{1}=\rho_{2}=\cdots=\rho_{n}=\rho$.

\subsubsection{Risk aggregation: variance-covariance approach}

By the variance-covariance approach, the aggregate capital $K$ at the corporate level is given by:

$$
K=\mathbb{E}[S]+\sqrt{\sum_{i=1}^{n} \sum_{j=1}^{n} r_{i j}\left(\rho\left(X_{i}\right)-\mathbb{E}\left[X_{i}\right]\right)\left(\rho\left(X_{j}\right)-\mathbb{E}\left[X_{j}\right]\right)},
$$

where $r_{i j}$, for $i, j=1,2, \ldots, n$, is the correlation coefficient between the losses $X_{i}$ and $X_{j}$ of the LOBs/risks. The classical variance-covariance approach for risk aggregation fails to capture non-linear dependence structures. To illustrate this sufficiently, assume that the risk measure $\rho$ has a positive risk loading, and thus is strictly greater than the expectation; moreover, assume that the risk measure $\rho$ is comonotonic additive and the losses $X_{1}, X_{2}, \ldots, X_{n}$ are comonotonic. By the square-root formula (6), together with the fact that $r_{i j} \leq 1$, for $i, j=1,2, \ldots, n$, the aggregate capital, by the variance-covariance approach,

$$
\begin{aligned}
K^{V} & =\mathbb{E}[S]+\sqrt{\sum_{i=1}^{n} \sum_{j=1}^{n} r_{i j}\left(\rho\left(X_{i}\right)-\mathbb{E}\left[X_{i}\right]\right)\left(\rho\left(X_{j}\right)-\mathbb{E}\left[X_{j}\right]\right)} \\
& \leq \mathbb{E}[S]+\sqrt{\left(\sum_{i=1}^{n}\left(\rho\left(X_{i}\right)-\mathbb{E}\left[X_{i}\right]\right)\right)^{2}}=\rho(S),
\end{aligned}
$$

where the last equality is due to the assumed comonotonic additivity of the risk measure $\rho$, and the assumption that the losses $X_{1}, X_{2}, \ldots, X_{n}$ are comonotonic; moreover, the total capital $K^{V}$ attains the upper bound $\rho(S)$ if and only if the correlation coefficients $r_{i j}=1$, for all $i, j=1,2, \ldots, n$.

Nevertheless, it is well-known that comonotonicity does not necessarily imply the correlation coefficients being 1, especially for non-elliptically distributed losses. See, for instance, McNeil et al. (2015). Indeed, the range of attainable correlation coefficients, $\left[r_{i j}^{\min }, r_{i j}^{\max }\right]$, is much narrower than the usual range $[-1,1]$, which is derived by the CauchySchwarz inequality. Moreover, the losses $X_{1}, X_{2}, \ldots, X_{n}$ are comonotonic if and only if the correlation coefficients $r_{i j}=r_{i j}^{\max }$, for all $i, j=1,2, \ldots, n$. Therefore, if the attainable 
upper bound $r_{i j}^{\max }$ is strictly less than 1 , the allocated aggregate capital $K^{V}$ cannot attain the upper bound $\rho(S)$, or, more precisely, is strictly less than the risk measure $\rho(S)$; hence, the classical variance-covariance approach does not provide a sufficient aggregate capital protection in terms of the risk measure $\rho(S)$; consequently, regardless of the subsequent capital allocation principle employed, it cannot provide a sufficient capital protection to some individual loss in terms of the risk measure $\rho\left(X_{i}\right)$, for $i=1,2, \ldots, n$, due to comonotonicity.

In contrast, under the same assumption that the risk measure $\rho$ is comonotonic additive and the losses $X_{1}, X_{2}, \ldots, X_{n}$ are comonotonic, the least squares holistic principle in (2) or (4) does provide a sufficient coverage of losses in terms of the risk measure $\rho(S)$. Indeed,

$$
K^{H}=\rho(S)+A^{\prime}\left(\sum_{r=1}^{n} \rho\left(X_{r}\right)-\rho(S)\right)=\rho(S) .
$$

To further illustrate this pitfall in the classical variance-covariance approach, consider the following numerical example, with $n=2$ and the risk measure $\rho=\mathrm{TVaR}_{0.99}$, which has a $99 \%$ confidence level. Assume that, marginally, $X_{1} \sim$ Pareto $\left(\kappa_{1}, \theta_{1}\right)$ and $X_{2} \sim$ Pareto $\left(\kappa_{2}, \theta_{2}\right)$. Then, their correlation coefficient $r_{12}$ is upper-bounded by an attainable $r_{12}^{\max }$, where

$$
r_{12}^{\max }=\frac{\sqrt{\left(1-\frac{2}{\kappa_{1}}\right)\left(1-\frac{2}{\kappa_{2}}\right)}}{1-\frac{1}{\kappa_{1}}-\frac{1}{\kappa_{2}}},
$$

which is attained when $X_{1}$ and $X_{2}$ are comonotonic. Assume that this is really the case, that $X_{1}$ and $X_{2}$ are comonotonic, and let $\kappa_{1}=4, \kappa_{2}=2, \theta_{1}=60$, and $\theta_{2}=10$. Consequently, $r_{12}=r_{12}^{\max }=0$; by the square-root formula (6), $K^{V}=280$, which only constitutes around $75 \%$ of the risk measure $\rho(S)=\mathrm{TVaR}_{0.99}(S)=383$. In contrast, the least squares holistic principle fully covers the risk measure: $K^{H}=\operatorname{TVaR}_{0.99}(S)=383$.

\subsubsection{Capital allocation: Euler allocation principle}

In this section, we will illustrate that the Euler allocation principle could suffer from a serious under/over-reserve issue, especially when the individual losses have an extremely negative dependence structure. To this end, assume that the risk measure $\rho=\mathrm{TVaR}_{\alpha}$, which has an $\alpha=95 \%$ confidence level, and the losses $X_{1}, X_{2}, \ldots, X_{n}$ are multivariate normally distributed. As shown in Panjer and Jing (2001) [see also (Feng 2018, Section 5.6)], for $i=1,2, \ldots, n$, the Euler allocation formula is given by

$$
K_{i}^{E}=\mathbb{E}\left[X_{i}\right]+\frac{\phi\left(\Phi^{-1}(\alpha)\right)}{1-\alpha} \frac{\sum_{j=1}^{n} r_{i j} \sigma\left(X_{i}\right) \sigma\left(X_{j}\right)}{\sqrt{\sum_{i=1}^{n} \sum_{j=1}^{n} r_{i j} \sigma\left(X_{i}\right) \sigma\left(X_{j}\right)}} .
$$

On the other hand, the least squares holistic principle in (3) or (5) is given by, for any $i=1,2, \ldots, n$,

$K_{i}^{H}=\mathbb{E}\left[X_{i}\right]+\frac{\phi\left(\Phi^{-1}(\alpha)\right)}{1-\alpha}\left(\sigma\left(X_{i}\right)-B_{i}^{\prime}\left(\sum_{r=1}^{n} \sigma\left(X_{r}\right)-\sqrt{\sum_{r=1}^{n} \sum_{j=1}^{n} r_{i j} \sigma\left(X_{r}\right) \sigma\left(X_{j}\right)}\right)\right)$.

In order to discuss almost-countermonotonic scenarios, consider the case that there are only two individual LOBs/risks, with $n=2$. Without loss of generality, assume that $\sigma\left(X_{1}\right) \geq$ $\sigma\left(X_{2}\right)$. Note the following two important observations regarding the Euler allocated capitals. 


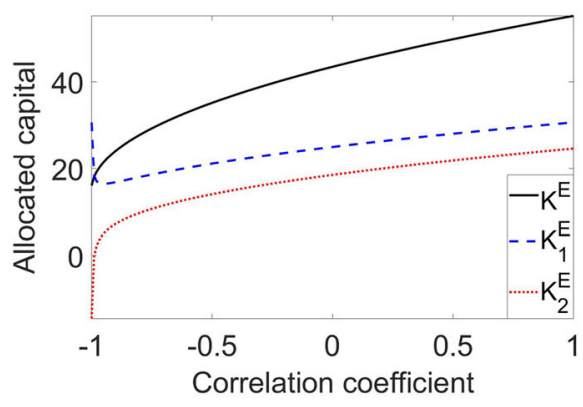

(a) Euler allocation principle

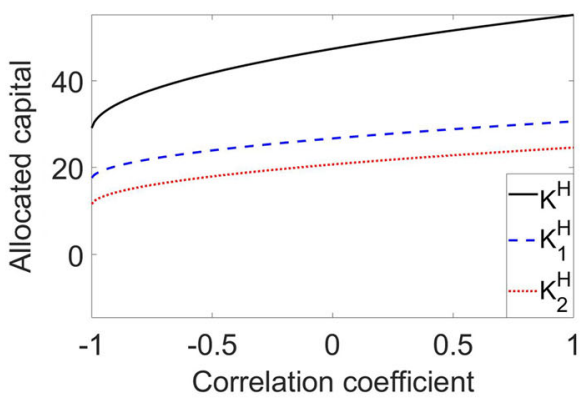

(b) Holistic principle

Fig. 8 Comparison between Euler and holistic principles with Tail Value-at-Risk measurements and $\left(X_{1}, X_{2}\right) \sim \operatorname{Normal}_{2}\left((10,5),\left[\begin{array}{cc}100 & 95 r_{12} \\ 95 r_{12} & 90.25\end{array}\right]\right)$

- [Stationary point $]$ It is easy to show that

$$
\frac{\mathrm{d} K_{1}^{E}}{\mathrm{~d} r_{12}}=\frac{\sigma\left(X_{1}\right) \sigma\left(X_{2}\right)^{2}\left(r_{12} \sigma\left(X_{1}\right)+\sigma\left(X_{2}\right)\right)}{\left(\sigma\left(X_{1}\right)^{2}+2 r_{12} \sigma\left(X_{1}\right) \sigma\left(X_{2}\right)+\sigma\left(X_{2}\right)^{2}\right)^{\frac{3}{2}}},
$$

which imples that $K_{1}^{E}$ decreases with $r_{12} \in\left[-1,-\sigma\left(X_{2}\right) / \sigma\left(X_{1}\right)\right]$ and increases with $r_{12} \in\left[-\sigma\left(X_{2}\right) / \sigma\left(X_{1}\right), 1\right]$. Therefore, the allocated capital $K_{1}^{E}$ to the first loss $X_{1}$, by the Euler allocation principle, has a stationary point at $r_{12}^{*}=-\sigma\left(X_{2}\right) / \sigma\left(X_{1}\right)$.

- [Negative allocated capital] Observe that

$$
\lim _{r_{12} \rightarrow-1} \frac{r_{12} \sigma\left(X_{1}\right) \sigma\left(X_{2}\right)+\sigma\left(X_{2}\right)^{2}}{\sqrt{\sigma\left(X_{1}\right)^{2}+2 r_{12} \sigma\left(X_{1}\right) \sigma\left(X_{2}\right)+\sigma\left(X_{2}\right)^{2}}}=-\sigma\left(X_{2}\right),
$$

and hence

$$
\lim _{r_{12} \rightarrow-1} K_{2}^{E}=\mathbb{E}\left[X_{2}\right]-\frac{\phi\left(\Phi^{-1}(\alpha)\right)}{1-\alpha} \sigma\left(X_{2}\right) .
$$

One can also show that

$$
\lim _{\alpha \rightarrow 1} \frac{\phi\left(\Phi^{-1}(\alpha)\right)}{1-\alpha}=-\left.\frac{\mathrm{d}}{\mathrm{d} \alpha} \phi\left(\Phi^{-1}(\alpha)\right)\right|_{\alpha=1}=\left.\Phi^{-1}(\alpha)\right|_{\alpha=1} .
$$

Therefore, the allocated capital $K_{2}^{E}$ to the second loss $X_{2}$, by the Euler allocation principle, becomes severely negative, when the confidence level is close to 1 and the correlation coefficient is close to -1 .

By combining these two results, the first line of business is over-reserved, while the second line is severely under-reserved, when the correlation coefficient between the two losses approaches -1 , with the chosen confidence level $\alpha=95 \%$, which is close enough to 1 .

To further illustrate this peculiar result with the Euler allocation principle, consider the following numerical example. Assume that $\mathbb{E}\left[X_{1}\right]=10, \mathbb{E}\left[X_{2}\right]=5, \sigma\left(X_{1}\right)=10$, and $\sigma\left(X_{2}\right)=9.5$. Moreover, assume that equal weights apply to the individual LOBs/risks and the corporate, with $\omega_{1}=\omega_{2}=\omega$. Figure 8a, b depict the allocated capital respectively, by the classical Euler allocation principle, and by the least squares holistic principle, for various correlation coefficients between the two losses. 
Figure 8a confirms the theoretical analysis above regarding the over/under-reserve issue, with respect to the risk measure $\operatorname{TVaR}_{\alpha}\left(X_{i}\right)$, for $i=1,2$. This result is due to the fact that Euler allocation principle recognizes the diversification benefit of these two losses and the allocated capital as a sensitive measure gives more to a risk or line of business that affects the aggregate more drastically. Such a sensitivity measure does not always serve the purpose of capital allocation, which is to ensure sufficient capital protection for each LOB/risk.

In contrast, as shown in Fig. 8b, the least squares holistic principle provides a more equitable distribution of the holistic aggregate capital between the two LOBs/risks, even in the cases with extreme negative dependence structure. As it takes into account all risk metrics of individual units and the corporate, the holistic principle offers a balanced approach to the use of capitals for all objectives set by the corporate management. Moreover, the least squares holistic principle offers a higher level of protection in terms of capital adequacy than the classical Euler allocation principle.

Finally, as shown in Fig. 8a, b , when the correlation coefficient $r_{12}$ reaches 1, the least squares holistic principle is the same as the classical Euler allocation principle. Indeed, since both losses $X_{1}$ and $X_{2}$ are continuous and comonotonic, the Euler allocation principle gives, for $i=1,2, \ldots, n$,

$$
K_{i}^{E}=\mathbb{E}\left[X_{i} \mid S \geq \operatorname{VaR}_{\alpha}(S)\right]=\mathbb{E}\left[X_{i} \mid X_{i} \geq \operatorname{VaR}_{\alpha}\left(X_{i}\right)\right]=\operatorname{TVaR}_{\alpha}\left(X_{i}\right),
$$

while the least squares holistic principle gives, for $i=1,2, \ldots, n$,

$$
K_{i}^{H}=\operatorname{TVaR}_{\alpha}\left(X_{i}\right)-B_{i}^{\prime}\left(\sum_{r=1}^{n} \operatorname{TVaR}_{\alpha}\left(X_{r}\right)-\operatorname{TVaR}_{\alpha}(S)\right)=\operatorname{TVaR}_{\alpha}\left(X_{i}\right) .
$$

\subsection{Holistic allocation implementation in a typical commercial bank}

This section is intended to show how the holistic principle can be applied to a real-business setting. The practice of risk aggregation and capital allocation is common for a financial conglomerate under regulatory requirement or for internal risk management purpose. A financial conglomerate can be divided into 4 different major lines of business, namely universal bank, life insurance company, property and casualty insurance company, non-licensed subsidiary (see for example Kuritzkes et al. 2003). Each line of business often carries a different risk profile. For example, credit (resp. market, insurance, operational) risk is a major source of uncertainty for universal bank (resp. life insurance company, property and casualty insurance company, non-licensed subsidiary).

Here we consider a stylized portfolio representative of a typical commercial bank with a small component of insurance activity, developed in Morone et al. (2007). The goal is to determine a total aggregated measure of diversified economic capital and to allocate the diversified economic capital back to different risk types. The time horizon of risks is set to one year and the economic capital requirement is assessed to protect losses at the $99.96 \%$ level. Morone et al. (2007) considered four macrocategories of nine risk types, including credit (performing loans and defaulted loans), market (interest rate risk in banking book, trading book, equity, property), operating (business and operational) and insurance (not subdivided any further).

The summary statistics of empirical data from all risk types are given in Table 3 . The case study in Morone et al. (2007) uses a meta-Gaussian framework in which six of the risks are modeled by Gaussian marginal distributions fitted to empirical distributions. The rest three risk types are not Gaussian distributed. The distribution of performing loans is 
determined by Monte Carlo simulation of loan portfolio asset values using Merton theory, the trading loss distribution is obtained through historical simulation, and the operational risk loss distribution is generated by an actuarial advanced measurement approach. Note that the operational risk loss distribution has large skewness and kurtosis, indicating significant likelihood of large losses. It is natural to expect that special consideration should be given to operational risk given its fat tail and sufficiently large capital is needed to prepare for severe cases. The dependence structure of individual risks is determined by the $t$-copula.

As shown in Table 3, standalone economic capitals are determined by VaRs at the confidence level $\alpha_{1}=\alpha_{2}=\cdots=\alpha_{9}=99.6 \%$. For simplicity, we ignore capital management objectives for this example, i.e., $v_{1}=v_{2}=\cdots=v_{9}=0$. Moreover, risk management objectives for all risks are treated equal, i.e., $\omega_{1}=\omega_{2}=\cdots=\omega_{9}$. Standalone capitals $K_{i}^{R}=\operatorname{VaR}_{99.6 \%}\left(X_{i}\right), i=1,2, \ldots, 9$, are given in the second last column of Table 3, with their sum given as $\sum_{i=1}^{n} K_{i}^{R}=10000$. Given marginal loss distributions and their dependency through $t$-copula, the optimal aggregate capital is given by $K^{R}=\operatorname{VaR}_{99.6 \%}(S)=8498$. These results are the same as in Table 6 of Morone et al. (2007). We employ the holistic principle shown in (3) or (5) for this example. Allocated capitals are summarized in the last column of Table 3, with the holistic aggregate capital given by $K^{H}=8648.2$. These findings are in line with the economic interpretation in Sect. 3.1.1 that the diversified capital, i.e., $\sum_{i=1}^{9} K_{i}^{R}-K^{R}=1502$, is distributed to each risk via the equal harmonic weighting.

\section{Constrained case}

There are circumstances in practice where allocated capitals need to be restricted. Regulators may be concerned with financial institutions gaming the regulatory system to their own advantages. For example, even if a regulator specifies the weights for all risk management and capital management objectives, a financial institution may be able to develop a sophisticated internal model to estimate loss distributions in such a way that the capitals are set artificially low. Therefore, in addition to requiring full disclosure of internal models, a regulator could impose floors on required capitals for different types of LOBs/risks that are determined by other methods. For example, one could use the factor based approach for floors, which are commonly used in current regulatory frameworks such as Solvency II, Risk-Based Capital, etc.

Throughout this section, we assume that the regulator imposes a minimum allocated capital requirement that $\underline{b}_{i} \leq K_{i}$ for $i=1,2, \ldots, n$. Consider also the case that the corporate has certain capital allocation budget constraint that $K_{i} \leq \bar{b}_{i}$ for $i=1,2, \ldots, n$. Both the floors $\underline{b}_{i}$ 's and the caps $\bar{b}_{i}$ 's are set by exogenous methods. This section illustrates the constrained case only for the targeted and least squares holistic principle, since similar results can be extended for the minimal and least squares holistic principle.

Problem 3 The constrained targeted and least squares holistic principle $\left(K_{1}^{H^{*}}, K_{2}^{H^{*}}\right.$, $\left.\ldots, K_{n}^{H^{*}}\right)$ solves

$$
\begin{aligned}
& \inf _{\left(K_{1}, K_{2}, \ldots, K_{n}\right) \in \mathbb{R}^{n}} \sum_{i=1}^{n} v_{i}\left(c_{i}-K_{i}\right)^{2}+\sum_{i=1}^{n} \omega_{i} \mathbb{E}\left[\left(X_{i}-K_{i}\right)^{2} h_{i}\left(X_{i}\right)\right]+v(c-K)^{2}+\omega \mathbb{E}\left[(S-K)^{2} h(S)\right], \\
& \text { subject to } \underline{b}_{i} \leq K_{i} \leq \bar{b}_{i}, \quad i=1,2, \ldots, n .
\end{aligned}
$$




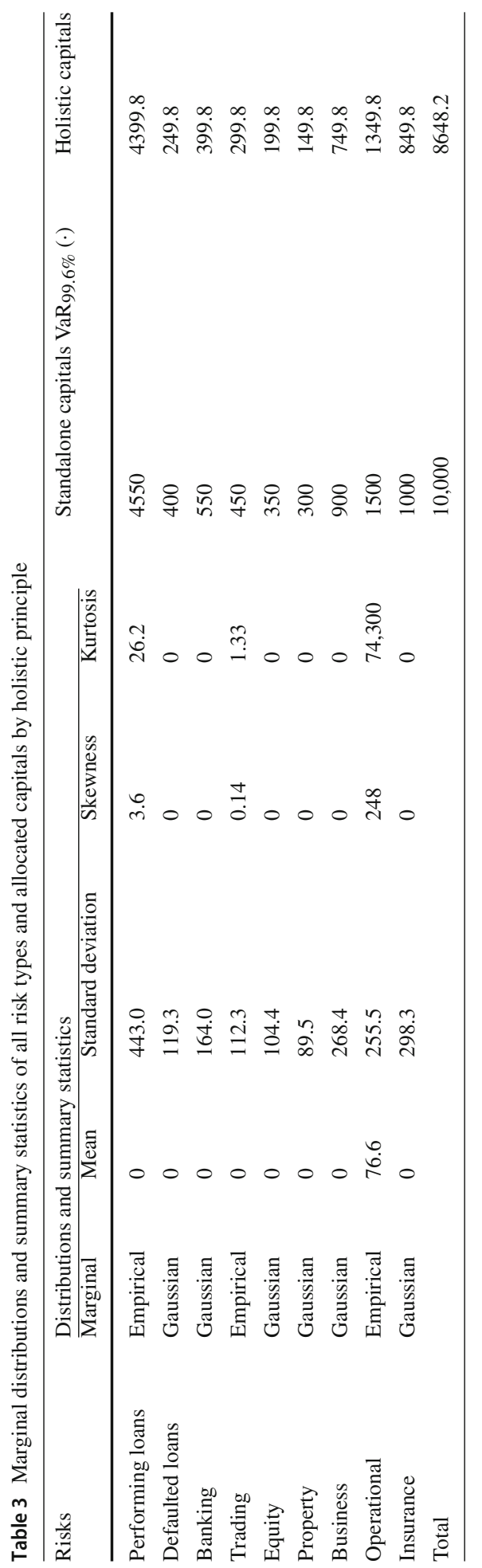


Table 4 Interpretations in unconstrained holistic allocated capital

\begin{tabular}{llll}
\hline Item & Interpretation & Item & Interpretation \\
\hline (1) & Allocated capital & (2) & Standalone capital \\
(3) & Allocation ratio & (4) & Sum of standalone capitals \\
(5) & Aggregate capital & (6) & Diversification benefit \\
\hline
\end{tabular}

For the constrained case, instead of first stating the result for Problem 3, as in Theorem 1 for Problem 1, we first provide economic interpretations in obtaining the constrained targeted and least squares holistic capitals in Theorem 4, which is to be shown below. Moreover, the special case with bivariate LOBs/risks prepares the embracing of the delicate Theorem 4.

\subsection{Economic interpretations}

The constrained targeted and least squares holistic aggregate and allocated capitals closely resemble those under the unconstrained case, in (2) and (3), and thus can also be interpreted in terms of horizontal and vertical balancings as illustrated in Fig. 5.

- [Horizontal balancing] Optimal aggregate and standalone capitals for the corporate and individual LOBs/risks are obtained in the same way as in the unconstrained case using the arithmetic weighting: for $i=1,2, \ldots, n, \bar{K}_{i}=\alpha_{i} K_{i}^{C}+\beta_{i} K_{i}^{R}$, and $\bar{K}=\alpha K^{C}+\beta K^{R}$.

- [Vertical balancing] Individual LOBs/risks, and the entire corporate, not only carry different objectives, but also have to juggle with capital constraints. It is not surprising that the analogy of the unconstrained holistic principle (3) is broken down into several cases for the constrained holistic principle.

For the ease of exposition, let us first recap the unconstrained holistic principle here. The unconstrained holistic aggregate capital is given by:

$$
K^{H}=\bar{K}+A\left(\sum_{r=1}^{n} \bar{K}_{r}-\bar{K}\right) \text {, }
$$

while, for $i=1,2, \ldots, n$, the unconstrained holistic allocated capital for the $i$ th LOB/risk is given by:

$$
\underbrace{K_{i}^{H}}_{\text {(1) }}=\underbrace{\bar{K}_{i}}_{\text {(2) }}-\underbrace{B_{i}}_{\text {(3) }}(\underbrace{\overbrace{\sum_{r=1}^{n} \bar{K}_{r}}^{\text {(6) }}-\underbrace{}_{\text {(5) }}}_{\text {(4) }}),
$$

where Table 4 recalls the interpretations of these six components.

For the constrained case, it turns out that the holistic principle considers the "balanced" capital for each LOB/risk and whether it conforms to the constraint. The "balanced" capital indicates the amount to be allocated, should there be no capital constraint for the LOB/risk under consideration. Mathematically, it is defined by, for $i=1,2, \ldots, n$, 


$$
\tilde{K}_{i}=\underbrace{\bar{K}_{i}}_{\text {(2) }}-\underbrace{\tilde{B}_{i}}_{\text {(3) }}(\underbrace{(\overbrace{\sum_{j=1, j \neq i}^{n}\left[\bar{K}_{j}\right]+\bar{K}_{i}}^{6}-\bar{K}}_{(4)}),
$$

where $[\cdot]$ is used to bound "balanced" capitals of other LOBs/risks by their respective constraints, i.e. $\left[\bar{K}_{j}\right]=\underline{b}_{j} \mathbb{1}_{\left\{\tilde{K}_{j}<\underline{b}_{j}\right\}}+\bar{K}_{j} \mathbb{1}_{\left\{\underline{b}_{j} \leq \tilde{K}_{j} \leq \bar{b}_{j}\right\}}+\bar{b}_{j} \mathbb{1}_{\left\{\bar{b}_{j}<\tilde{K}_{j}\right\}}$; moreover, the allocation ratio $\tilde{B}_{i}$ for the $i$ th $\mathrm{LOB} /$ risk has a similar structure as $B_{i}$ in the unconstrained case, which herein is determined by a harmonic weighting:

$$
\tilde{B}_{i}=\frac{\frac{1}{v_{i}+\omega_{i}}}{\sum_{j=1, j \neq i}^{n} \frac{1}{v_{j}+\omega_{j}} \mathbb{1}_{\left\{\underline{b}_{j} \leq \tilde{K}_{j} \leq \bar{b}_{j}\right\}}+\frac{1}{v_{i}+\omega_{i}}+\frac{1}{v+\omega}} .
$$

Therefore, when the desired "balanced" capital of the $j$ th LOB/risk conforms to its constraint, its corresponding weight $1 /\left(v_{j}+\omega_{j}\right)$ is taken into account in the harmonic weighting, and its optimal standalone capital $\bar{K}_{j}$ is taken into consideration for diversification. However, when "balanced" capital falls out of its limits, the weight is not included in the harmonic weighting, and its optimal standalone capital is not taken into consideration for diversification; this is because the allocated capital for this LOB/risk is simply a bound of its capital constraint, and this LOB/risk cannot receive any extra distribution of remaining diversification benefit.

Finally, once we check whether all these "balanced" capitals conform to their respective constraints, we can determine the holistic allocated capitals by, for $i=1,2, \ldots, n$,

$$
K_{i}^{H^{*}}=\underline{b}_{i} \mathbb{1}_{\left\{\tilde{K}_{i}<\underline{b}_{i}\right\}}+\tilde{K}_{i} \mathbb{1}_{\left\{\underline{b}_{i} \leq \tilde{K}_{i} \leq \bar{b}_{i}\right\}}+\bar{b}_{i} \mathbb{1}_{\left\{\bar{b}_{i}<\tilde{K}_{i}\right\}} .
$$

With analogy to (2) the constrained holistic aggregate capital is given by:

$$
K^{H^{*}}=\sum_{i=1}^{n} K_{i}^{H^{*}}=\bar{K}+\tilde{A}\left(\sum_{j=1}^{n}\left[\bar{K}_{j}\right]-\bar{K}\right),
$$

where the allocation ratio $\tilde{A}$ is given by

$$
\tilde{A}=\frac{\frac{1}{v+\omega}}{\sum_{j=1}^{n} \frac{1}{v_{j}+\omega_{j}} \mathbb{1}_{\left\{\underline{b}_{j} \leq \tilde{K}_{j} \leq \bar{b}_{j}\right\}}+\frac{1}{v+\omega}} .
$$

Recall that, in the unconstrained case, $A+\sum_{j=1}^{n} B_{j}=1$, which indicates that the diversification benefit (item (6)) is fully attributable to all components in the corporate hierarchy. We can expect a parallel result where the diversification benefit is full explained by elements in this constrained balancing. Since some LOBs/risks are confined by capital limits, the allocation of diversification benefit only goes to those which are free within their capital limits to receive benefits. Therefore, $\tilde{A}+\sum_{j=1}^{n} \tilde{B}_{j} \mathbb{1}_{\left\{\underline{b}_{j} \leq \tilde{K}_{j} \leq \bar{b}_{j}\right\}}=1$.

\subsection{Bivariate risks or lines of business}

The balancing acts with capital constraints is best illustrated with the case of two LOBs/risks. Depending on where the "balanced" capitals $\tilde{K}_{1}$ and $\tilde{K}_{2}$ fall, there are a total of 9 regions 
Fig. 9 Partition by $\left(\tilde{K}_{1}, \tilde{K}_{2}\right)$

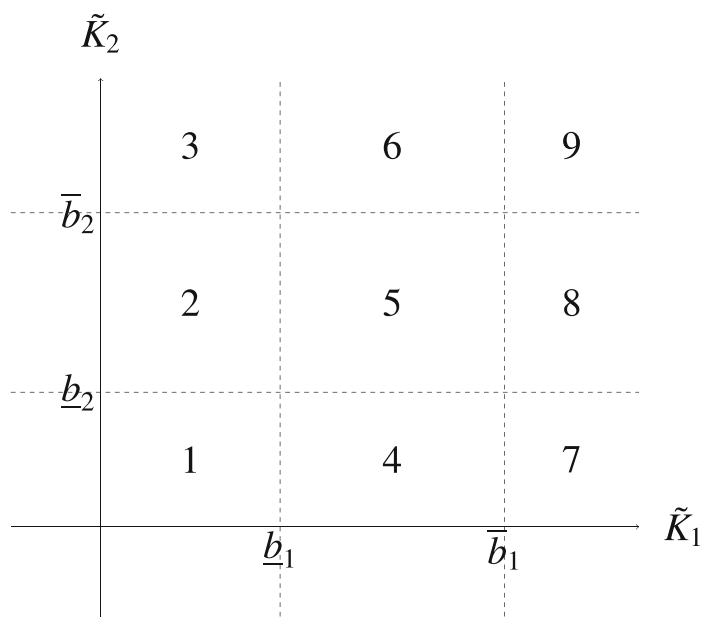

Fig. 10 Partition by $\left(\bar{K}_{1}, \bar{K}_{2}\right)$

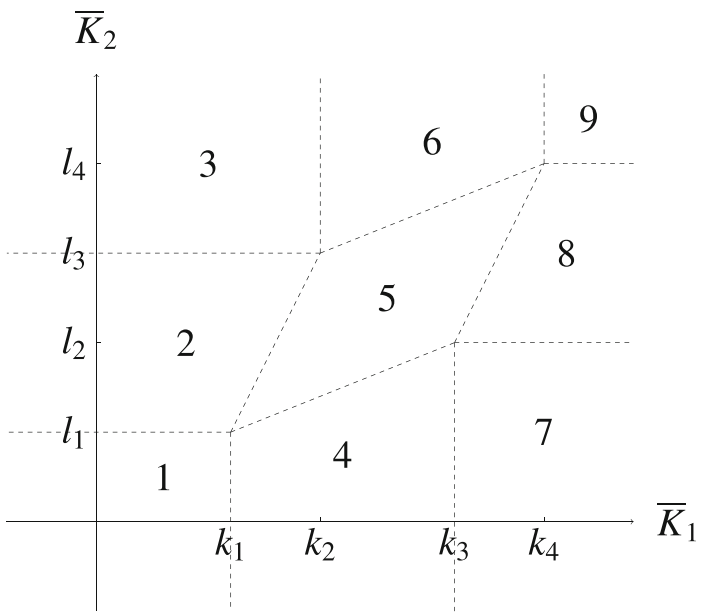

to be considered, as shown in Fig. 9. The conditions for all cases are spelled out in Table 5. Keep in mind that all optimal standalone capitals are determined in the horizontal balancing step and used in setting conditions for the vertical balancing step. In particular, in case 5, all weights $A, B_{1}$, and $B_{2}$ are the same as those in the unconstrained case, with $A+B_{1}+B_{2}=1$. On the other hand, the weights for other cases, $A_{1}^{*}, A_{2}^{*}, B_{1}^{*}$, and $B_{2}^{*}$ are defined as follows:

$A_{1}^{*}=\frac{\frac{1}{v+\omega}}{\frac{1}{v+\omega}+\frac{1}{v_{1}+\omega_{1}}}, \quad A_{2}^{*}=\frac{\frac{1}{v+\omega}}{\frac{1}{v+\omega}+\frac{1}{v_{2}+\omega_{2}}}, \quad B_{1}^{*}=\frac{\frac{1}{v_{1}+\omega_{1}}}{\frac{1}{v+\omega}+\frac{1}{v_{1}+\omega_{1}}}, \quad B_{2}^{*}=\frac{\frac{1}{v_{2}+\omega_{2}}}{\frac{1}{v+\omega}+\frac{1}{v_{2}+\omega_{2}}}$, and it is clear that $A_{1}^{*}+B_{1}^{*}=1$ and $A_{2}^{*}+B_{2}^{*}=1$.

It is also possible to represent these 9 cases in terms of the LOBs/risks' optimal standalone capitals, as shown in Fig. 10. The boundaries for optimal standalone capitals are given by

$$
\begin{array}{ll}
k_{1}=\underline{b}_{1}+\frac{v+\omega}{v_{1}+\omega}\left(\underline{b}_{1}+\underline{b}_{2}-\bar{K}\right), & l_{1}=\underline{b}_{2}+\frac{v+\omega}{v_{2}+\omega_{2}}\left(\underline{b}_{1}+\underline{b}_{2}-\bar{K}\right), \\
k_{2}=\underline{b}_{1}+\frac{v+\omega}{v_{1}+\omega_{1}}\left(\underline{b}_{1}+\bar{b}_{2}-\bar{K}\right), & l_{2}=\underline{b}_{2}+\frac{v+\omega}{v_{2}+\omega_{2}}\left(\bar{b}_{1}+\underline{b}_{2}-\bar{K}\right),
\end{array}
$$




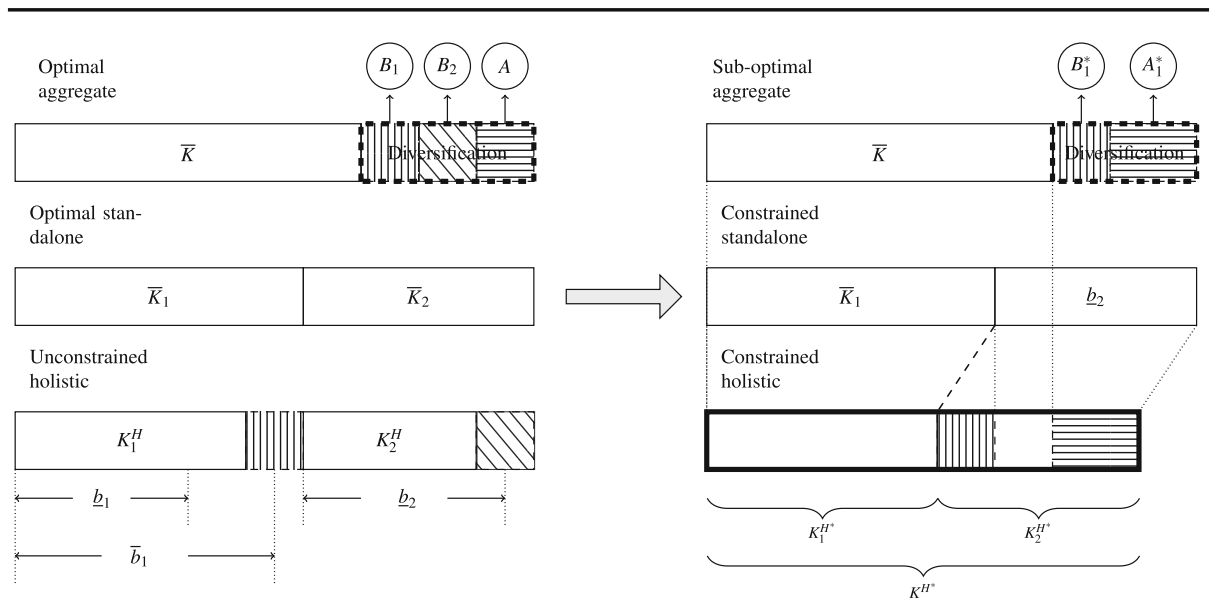

Fig. 11 Constrained holistic capitals for bivariate LOBs/risks-diversification benefit

$$
\begin{array}{ll}
k_{3}=\bar{b}_{1}+\frac{v+\omega}{v_{1}+\omega_{1}}\left(\bar{b}_{1}+\underline{b}_{2}-\bar{K}\right), & l_{3}=\bar{b}_{2}+\frac{v+\omega}{v_{2}+\omega_{2}}\left(\underline{b}_{1}+\bar{b}_{2}-\bar{K}\right), \\
k_{4}=\bar{b}_{1}+\frac{v+\omega}{v_{1}+\omega_{1}}\left(\bar{b}_{1}+\bar{b}_{2}-\bar{K}\right), & l_{4}=\bar{b}_{2}+\frac{v+\omega}{v_{2}+\omega_{2}}\left(\bar{b}_{1}+\bar{b}_{2}-\bar{K}\right) .
\end{array}
$$

Observe that, if the corporate goal is ignored, i.e. $v+\omega=0$, then the two divisions in Figs. 9 and 10 coincide with each other. Finally, the constrained holistic aggregate and allocated capitals for all cases are shown in Table 5, when there are two LOBs/risks.

To further understand why the vertical balancing is different from that in the unconstrained case, we show for example the reasoning behind the region 4 in Table 5. In the absence of any capital constraint, one would proceed to allocate capitals in the same way as in region 5. The left panel of Fig. 11 illustrates the unconstrained principle. Note that the top two bars show optimal aggregate capital $\bar{K}$, the sum of optimal standalone capitals $\bar{K}_{1}+\bar{K}_{2}$, and their difference being the diversification benefit. While the unconstrained holistic principle allocates capital for the first LOB/risk that satisfies the constraint that $\underline{b}_{1} \leq K_{1}^{H} \leq \bar{b}_{1}$, its allocated capital to the second LOB/risk does not, since $K_{2}^{H}<\underline{b}_{2}$. Therefore, such a first attempt of balancing fails to deliver an acceptable solution. Noting that the optimal standalone capital is unattainable, our second attempt is to fix the allocated capital for the second LOB/risk at the floor $\underline{b}_{2}$. The difference between the optimal aggregate capital $\bar{K}$, and the sum of the optimal standalone capital $\bar{K}_{1}$ and the fixed allocated capital $\underline{b}_{2}$, is clearly smaller than the diversification benefit in the unconstrained case. The advantage of the holistic principle is its ability to allocate the diversification benefit in a meaningful way while maintaining the Pareto optimality. Given that there is no room for further improvement for the second LOB/risk, the diversification benefit is to be redistributed only between the corporate and the first LOB/risk. Keep in mind that we no longer consider the second LOB/risk in the harmonic weighting $\left(B_{1}^{*}\right.$ and $A_{1}^{*}$ ) as it does not participate in the distribution of diversification benefit. By splitting the diversification benefit between the first LOB/risk and the corporate, we could add a portion of the diversification amount to the corporate's optimal aggregate capital and subtract the remaining portion from the first LOB/risk's optimal standalone capital. The procedure for the second attempt of allocation is shown in the right panel of Fig. 11. In this case, capital constraints for both LOBs/risks are satisfied, and hence we reach an acceptable solution, which is also presented as case 4 in Table 5. 


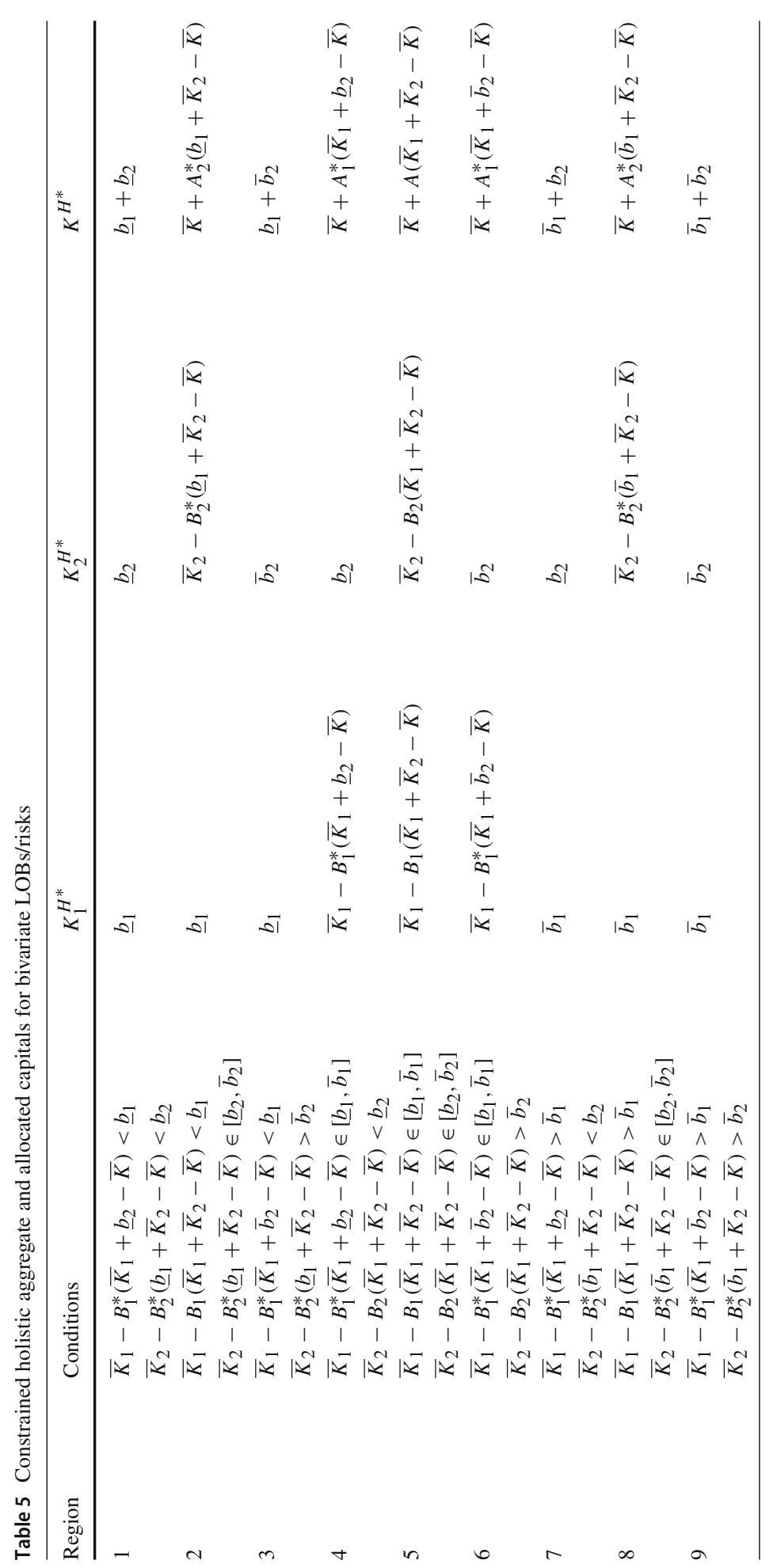




\subsection{General result}

While the economic interpretations are easy to explain, they are not obvious to implement by an algorithm. Hence, we state the following general result for any arbitrary number of LOBs/risks. The following result is a multivariate extension of the bivariate case listed in Table 5.

Theorem 4 Let $\mathcal{P}$ be a partition of $\mathbb{R}^{n}$, which is defined by

$$
\mathcal{P}:=\left\{I_{1} \times I_{2} \times \cdots \times I_{n}: I_{i}=\left(-\infty, \underline{b}_{i}\right),\left[\underline{b}_{i}, \bar{b}_{i}\right] \text {, or }\left(\bar{b}_{i}, \infty\right) \text { for } i=1,2, \ldots, n\right\} .
$$

For any $i=1,2, \ldots, n$ and $\mathbf{I} \in \mathcal{P}$, define the diversification factor $\mathcal{B}_{i}$,

$$
\mathcal{B}_{i}(\mathbf{I}):=\frac{\frac{1}{v_{i}+\omega_{i}}}{\sum_{j=1, j \neq i}^{n} \mathcal{T}_{1}\left(I_{j}\right)+\frac{1}{v_{i}+\omega_{i}}+\frac{1}{v+\omega}}, \quad \mathcal{T}_{1}\left(I_{i}\right):=\frac{1}{v_{i}+\omega_{i}} \mathbb{1}_{\left\{I_{i}=\left[\underline{b}_{i}, \bar{b}_{i}\right]\right\}} .
$$

Consider a fixed $\mathbf{I} \in \mathcal{P}$. If, for any $i=1,2, \ldots, n$,

$$
\bar{K}_{i}-\mathcal{B}_{i}(\mathbf{I})\left(\sum_{j=1, j \neq i}^{n} \mathcal{T}_{2}\left(I_{j}\right)+\bar{K}_{i}-\bar{K}\right) \in I_{i}
$$

where $\mathcal{T}_{2}\left(I_{i}\right):=\underline{b}_{i} \mathbb{1}_{\left\{I_{i}=\left(-\infty, \underline{b}_{i}\right)\right\}}+\bar{K}_{i} \mathbb{1}_{\left\{I_{i}=\left[\underline{b}_{i}, \bar{b}_{i}\right]\right\}}+\bar{b}_{i} \mathbb{1}_{\left\{I_{i}=\left(\bar{b}_{i}, \infty\right)\right\}}$, then the constrained holistic principle $\left(K_{1}^{H^{*}}, K_{2}^{H^{*}}, \ldots, K_{n}^{H^{*}}\right)$, in Problem 3 , is given by, for any $i=1,2, \ldots, n$,

$$
K_{i}^{H^{*}}= \begin{cases}\underline{b}_{i}, & \text { if } I_{i}=\left(-\infty, \underline{b}_{i}\right) \\ \bar{K}_{i}-\mathcal{B}_{i}(\mathbf{I})\left(\sum_{j=1, j \neq 1}^{n} \mathcal{T}_{2}\left(I_{j}\right)+\bar{K}_{i}-\bar{K}\right), & \text { if } I_{i}=\left[\underline{b}_{i}, \bar{b}_{i}\right] \\ \bar{b}_{i}, & \text { if } I_{i}=\left(\bar{b}_{i}, \infty\right)\end{cases}
$$

\section{Concluding remarks}

Risk aggregation and capital allocation are complex issues for any large capitalization financial institution. There are many aspects of managerial considerations that build into the decision making with regard to capital setting. Most of existing risk aggregation and capital allocation methods were developed as separate matters, even though they are both pieces of the same puzzle_-risk and capital management framework. This paper introduces a holistic approach for a capital management system, which takes into account several important considerations. (1) Capital setting should be tied to specific managerial objectives, such as risk management, capital management, etc. Because the principle is proposed as a solution to an optimization problem, one can clearly identify the combination of all managerial objectives. (2) There should be a fair and transparent policy on various components of consideration. For example, the management could define how much weight each objective carries for capital setting. (3) The capitals should reflect the healthy competition among various units. The holistic principle offers a clear balancing of competing objectives for all components and the whole. Although this paper studies holistic principle with two levels of the corporate hierarchy, it can be extended to any hierarchy with more tiers in line with regulation, such as 
Solvency II standard (see, for example, Baione et al. 2020), and this will be investigated in future work.

Funding The research of Wing Fung Chong is supported by start-up funds provided by the Department of Mathematics and Department of Statistics, University of Illinois at Urbana-Champaign. Runhuan Feng acknowledges the support of the State Farm Companies Foundation Endowment. This research is also partially funded by a Center of Actuarial Excellence (CAE) Research Grant (2019-2021) from the Society of Actuaries.

\section{Compliance with ethical standards}

Conflicts of interest The authors declare that they have no conflict of interest.

Open Access This article is licensed under a Creative Commons Attribution 4.0 International License, which permits use, sharing, adaptation, distribution and reproduction in any medium or format, as long as you give appropriate credit to the original author(s) and the source, provide a link to the Creative Commons licence, and indicate if changes were made. The images or other third party material in this article are included in the article's Creative Commons licence, unless indicated otherwise in a credit line to the material. If material is not included in the article's Creative Commons licence and your intended use is not permitted by statutory regulation or exceeds the permitted use, you will need to obtain permission directly from the copyright holder. To view a copy of this licence, visit http://creativecommons.org/licenses/by/4.0/.

\section{Appendix A Proof of Theorem 3.5}

With the assumed capital allocation targets, the holistic allocated capitals in (5) can be rewritten as, for any $i=1,2, \ldots, n$ and $t=1,2, \ldots$,

$$
K_{i}^{H}(t)=\left(\alpha_{i}+\alpha B_{i}-\alpha_{i} B_{i}\right) K_{i}^{H}(t-1)+\sum_{r \neq i} B_{i}\left(\alpha-\alpha_{r}\right) K_{r}^{H}(t-1)+d_{i},
$$

where $d_{i}=\beta_{i} K_{i}^{R}-B_{i}\left(\sum_{r=1}^{n} \beta_{r} K_{r}^{R}-\beta K^{R}\right)$, in which all constants $\alpha_{i}, \beta_{i}, B_{i}$, for $i=1,2, \ldots, n, \alpha$, and $\beta$ are defined in Theorem 3.1. Recursively, for any $t=1,2, \ldots$, $\mathbf{K}^{H}(t)=M \mathbf{K}^{H}(t-1)+\mathbf{d}$, where $\mathbf{K}^{H}(t)=\left(K_{1}^{H}(t), K_{2}^{H}(t), \ldots, K_{n}^{H}(t)\right)^{\mathrm{tr}}, \mathbf{d}=$ $\left(d_{1}, d_{2}, \ldots, d_{n}\right)^{\text {tr }}$, and

$$
M=\left[\begin{array}{cccc}
\alpha_{1}+\alpha B_{1}-\alpha_{1} B_{1} & B_{1}\left(\alpha-\alpha_{2}\right) & \ldots & B_{1}\left(\alpha-\alpha_{n}\right) \\
B_{2}\left(\alpha-\alpha_{1}\right) & \alpha_{2}+\alpha B_{2}-\alpha_{2} B_{2} & \ldots & B_{2}\left(\alpha-\alpha_{n}\right) \\
\vdots & \vdots & \ddots & \vdots \\
B_{n}\left(\alpha-\alpha_{1}\right) & B_{n}\left(\alpha-\alpha_{2}\right) & \ldots & \alpha_{n}+\alpha B_{n}-\alpha_{n} B_{n}
\end{array}\right]
$$

Equivalently, for any $t=0,1,2, \ldots, \mathbf{K}^{H}(t)=M^{t} \mathbf{K}^{H}(0)+\sum_{l=0}^{t-1} M^{l} \mathbf{d}$. One can easily show that

$$
\lim _{t \rightarrow \infty} M^{t}=0 \text { (zero matrix), } \quad \lim _{t \rightarrow \infty} \sum_{l=0}^{t-1} M^{l}=(I-M)^{-1},
$$

and hence $\lim _{t \rightarrow \infty} \mathbf{K}^{H}(t)=(I-M)^{-1} \mathbf{d}$, which is independent of $\mathbf{K}^{H}(0)$. 


\section{Appendix B Proof of Theorem 4.1}

Define the Lagrangian function, for any $K_{i}, \lambda_{i}, \eta_{i} \in \mathbb{R}$, for $i=1,2, \ldots, n$,

$$
\begin{aligned}
L & \left(K_{1}, K_{2}, \ldots, K_{n} ; \lambda_{1}, \lambda_{2}, \ldots, \lambda_{n}, \eta_{1}, \eta_{2}, \ldots, \eta_{n}\right) \\
= & \sum_{i=1}^{n} v_{i}\left(c_{i}-K_{i}\right)^{2}+\sum_{i=1}^{n} \omega_{i} \mathbb{E}\left[\left(X_{i}-K_{i}\right)^{2} h_{i}\left(X_{i}\right)\right]+v(c-K)^{2}+\omega \mathbb{E}\left[(S-K)^{2} h(S)\right] \\
& +\sum_{i=1}^{n} \lambda_{i}\left(\underline{b}_{i}-K_{i}\right)+\sum_{i=1}^{n} \eta_{i}\left(K_{i}-\bar{b}_{i}\right) .
\end{aligned}
$$

By the Karush-Kuhn-Tucker conditions, the optimal capital allocations, together with optimal Lagrange multipliers, satisfy, for any $i=1,2, \ldots, n$,

$$
\frac{\partial L^{H^{*}}}{\partial K_{i}}=0, \quad \underline{b}_{i} \leq K_{i}^{H^{*}} \leq \bar{b}_{i}, \quad \lambda_{i}^{H^{*}}, \eta_{i}^{H^{*}} \geq 0, \quad \lambda_{i}^{H^{*}}\left(\underline{b}_{i}-K_{i}^{H^{*}}\right)=0, \quad \eta_{i}^{H^{*}}\left(K_{i}^{H^{*}}-\bar{b}_{i}\right)=0,
$$

where the arguments for partial derivatives are omitted for notational simplicity, while $L^{H^{*}}$ denotes the Lagrangian function value with the optimal capital allocations, $K_{1}^{H^{*}}, K_{2}^{H^{*}}, \ldots$, $K_{n}^{H^{*}}$, and the optimal Lagrange multipliers, $\lambda_{1}^{H^{*}}, \lambda_{2}^{H^{*}}, \ldots, \lambda_{n}^{H^{*}}, \eta_{1}^{H^{*}}, \eta_{2}^{H^{*}}, \ldots, \eta_{n}^{H^{*}}$; the first order conditions of the Lagrange function are given by, for any $i=1,2, \ldots, n$,

$$
s_{i}\left(K_{1}^{H^{*}}, K_{2}^{H^{*}}, \ldots, K_{n}^{H^{*}}\right)-\lambda_{i}^{H^{*}}+\eta_{i}^{H^{*}}=0,
$$

where for any $K_{1}, K_{2}, \ldots, K_{n} \in \mathbb{R}$,

$$
s_{i}\left(K_{1}, K_{2}, \ldots, K_{n}\right)=2\left(v_{i}+\omega_{i}+v+\omega\right) K_{i}+2(v+\omega) \sum_{r \neq i} K_{r}-2\left(\left(v_{i}+\omega_{i}\right) \bar{K}_{i}+(v+\omega) \bar{K}\right) .
$$

Let $i=1,2, \ldots, n$. Denote $\tilde{K}_{i}$ be the solution of the equation

$$
s_{i}\left(K_{1}^{H^{*}}, K_{2}^{H^{*}}, \ldots, K_{i-1}^{H^{*}}, K_{i}, K_{i+1}^{H^{*}}, \ldots, K_{n}^{H^{*}}\right)=0 .
$$

If $\underline{b}_{i} \leq \tilde{K}_{i} \leq \bar{b}_{i}$, then $K_{i}^{H^{*}}=\tilde{K}_{i}$, and $\lambda_{i}^{H^{*}}=\eta_{i}^{H^{*}}=0$. If $\tilde{K}_{i}<\underline{b}_{i}$, then $K_{i}^{H^{*}}=\underline{b}_{i}$, $\eta_{i}^{H^{*}}=0$, and

$$
\lambda_{i}^{H^{*}}=s_{i}\left(K_{1}^{H^{*}}, K_{2}^{H^{*}}, \ldots, K_{i-1}^{H^{*}}, K_{i}^{H^{*}}, K_{i+1}^{H^{*}}, \ldots, K_{n}^{H^{*}}\right),
$$

which is non-negative; indeed,

$\lambda_{i}^{H^{*}} \geq 2\left(v_{i}+\omega_{i}+v+\omega\right) \tilde{K}_{i}+2(v+\omega) \sum_{r \neq i} K_{r}^{H^{*}}-2\left(\left(v_{i}+\omega_{i}\right) \bar{K}_{i}+(v+\omega) \bar{K}\right)=0$.

If $\bar{b}_{i}<\tilde{K}_{i}$, then $K_{i}^{H^{*}}=\bar{b}_{i}, \lambda_{i}^{H^{*}}=0$, and

$$
\eta_{i}^{H^{*}}=-s_{i}\left(K_{1}^{H^{*}}, K_{2}^{H^{*}}, \ldots, K_{i-1}^{H^{*}}, K_{i}^{H^{*}}, K_{i+1}^{H^{*}}, \ldots, K_{n}^{H^{*}}\right),
$$

which can be shown to be non-negative by similar arguments.

Therefore, the optimal capital allocations, $K_{1}^{H^{*}}, K_{2}^{H^{*}}, \ldots, K_{n}^{H^{*}}$, are given by, for any $i=1,2, \ldots, n$,

$$
K_{i}^{H^{*}}=\underline{b}_{i} \mathbb{1}_{\left\{\tilde{K}_{i}<\underline{b}_{i}\right\}}+\tilde{K}_{i} \mathbb{1}_{\left\{\underline{b}_{i} \leq \tilde{K}_{i} \leq \bar{b}_{i}\right\}}+\bar{b}_{i} \mathbb{1}_{\left\{\bar{b}_{i}<\tilde{K}_{i}\right\}},
$$


where $\tilde{K}_{1}, \tilde{K}_{2}, \ldots, \tilde{K}_{n}$ solve the following system of semi-linear equations: for any $i=$ $1,2, \ldots, n$,

$$
\begin{aligned}
& \left(v_{i}+\omega_{i}+v+\omega\right) \tilde{K}_{i}+(v+\omega) \sum_{r \neq i}\left(\underline{b}_{r} \mathbb{1}_{\left\{\tilde{K}_{r}<\underline{b}_{r}\right\}}+\tilde{K}_{r} \mathbb{1}_{\left\{\underline{b}_{r} \leq \tilde{K}_{r} \leq \bar{b}_{r}\right\}}+\bar{b}_{r} \mathbb{1}_{\left\{\bar{b}_{r}<\tilde{K}_{r}\right\}}\right) \\
& -\left(\left(v_{i}+\omega_{i}\right) \bar{K}_{i}+(v+\omega) \bar{K}\right)=0 .
\end{aligned}
$$

Fix an $\mathbf{I} \in \mathcal{P}$. Firstly, assume that $\mathbf{I}$ is ordered such that there exist $j=0,1, \ldots, n$ and $k=j+1, \ldots, n$ such that, for any $r=1,2, \ldots, j, I_{r}=\left(-\infty, \underline{b}_{r}\right)$; for any $r=$ $j+1, j+2, \ldots, k, I_{r}=\left[\underline{b}_{r}, \bar{b}_{r}\right]$; and, for any $r=k+1, k+2, \ldots, n, I_{r}=\left(\bar{b}_{r}, \infty\right)$. Suppose that, for any $i=1,2, \ldots, n$, condition (8) holds.

To solve the system of semi-linear equations (9), consider the following two cases.

- Case 1: Suppose that, for any $r=1,2, \ldots, j, \tilde{K}_{r}<\underline{b}_{r}$; for any $r=j+1, j+2, \ldots, k$, $\underline{b}_{r} \leq \tilde{K}_{r} \leq \bar{b}_{r} ;$ and, for any $r=k+1, k+2, \ldots, n, \bar{b}_{r}<\tilde{K}_{r}$. In this case, $\tilde{K}_{1}, \tilde{K}_{2}, \ldots, \tilde{K}_{n}$ are solved analytically from the corresponding system of linear equations, which are given by, for any $i=1,2, \ldots, n$, the left-hand-side in condition (8). Therefore, by the conditions (8), $\tilde{K}_{1}, \tilde{K}_{2}, \ldots, \tilde{K}_{n}$ solve the system of semi-linear equations (9).

- Case 2: Suppose that any one of the inequalities in case 1 does not hold. Then the $\tilde{K}_{1}, \tilde{K}_{2}, \ldots, \tilde{K}_{n}$, solved from the corresponding system of linear equations, do not satisfy the conditions (8), and hence do not solve the system of semi-linear equations (9).

Hence, the solution $\tilde{K}_{1}, \tilde{K}_{2}, \ldots, \tilde{K}_{n}$ of the system of semi-linear equations (9) satisfy that, for any $i=1,2, \ldots, j, \tilde{K}_{i}<\underline{b}_{i}$; for any $i=j+1, j+2, \ldots, k, \underline{b}_{i} \leq \tilde{K}_{i} \leq \bar{b}_{i}$; and, for any $i=k+1, k+2, \ldots, n, \bar{b}_{i}<\tilde{K}_{i}$. By (8), for any $i=1,2, \ldots, j, K_{i}^{H^{*}}=\underline{b}_{i}$; for any $i=j+1, j+2, \ldots, k$,

$$
K_{i}^{H^{*}}=\bar{K}_{i}-\frac{\frac{1}{v_{i}+\omega_{i}}}{\sum_{r=j+1, r \neq i}^{k} \frac{1}{v_{r}+\omega_{r}}+\frac{1}{v_{i}+\omega_{i}}+\frac{1}{v+\omega}}\left(\sum_{r=1}^{j} \underline{b}_{r}+\sum_{r=j+1, r \neq i}^{k} \bar{K}_{r}+\sum_{r=k+1}^{n} \bar{b}_{r}+\bar{K}_{i}-\bar{K}\right) ;
$$

and, for any $i=k+1, k+2, \ldots, n, K_{i}^{H^{*}}=\bar{b}_{i}$.

Finally, any $\mathbf{I} \in \mathcal{P}$ with unordered intervals can be first ordered by a permutation $\Pi$; then, the above arguments are applied to obtain $K_{\Pi(1)}^{H^{*}}, K_{\Pi(2)}^{H^{*}}, \ldots, K_{\Pi(n)}^{H^{*}}$; the optimal capital allocations, $K_{1}^{H^{*}}, K_{2}^{H^{*}}, \ldots, K_{n}^{H^{*}}$, are finally obtained by taking the permutation inverse $\Pi^{-1}$ on $K_{\Pi(1)}^{H^{*}}, K_{\Pi(2)}^{H^{*}}, \ldots, K_{\Pi(n)}^{H^{*}}$.

\section{References}

Anderson, J. J., \& Thompson, H. E. (1971). Financial implications of over-reserving in nonlife insurance companies. Journal of Risk and Insurance, 38(3), 333-342. https://doi.org/10.2307/251398.

Arbenz, P., Hummel, C., \& Mainik, G. (2012). Copula based hierarchical risk aggregation through sample reordering. Insurance: Mathematics and Economics, 51(1), 122-133. https://doi.org/10.1016/j. insmatheco.2012.03.009.

Asimit, V., Peng, L., Wang, R., \& Yu, A. (2019). An efficient approach to quantile capital allocation and sensitivity analysis. Mathematical Finance, 29(4), 1131-1156. https://doi.org/10.1111/mafi.12211.

Baione, F., De Angelis, P., \& Granito, I. (2020). Capital allocation and RORAC optimization under solvency 2 standard formula. Annals of Operations Research. https://doi.org/10.1007/s10479-020-03543-6.

Bauer, D., \& Zanjani, G. (2014). The marginal cost of risk in a multi-period risk model (Tech. Rep.). Atlanta, GA: Robinson College of Business, Georgia State University. 
Bauer, D., \& Zanjani, G. (2016). The marginal cost of risk, risk measures, and capital allocation. Management Science, 62(5), 1431-1457. https://doi.org/10.1287/mnsc.2015.2190.

Bernard, C., Jiang, X., \& Wang, R. (2014). Risk aggregation with dependence uncertainty. Insurance: Mathematics and Economics, 54, 93-108. https://doi.org/10.1016/j.insmatheco.2013.11.005.

Biagini, F., Fouque, J. P., Frittelli, M., \& Meyer-Brandis, T. (2019). A unified approach to systemic risk measures via acceptance sets. Mathematical Finance, 29(1), 329-367. https://doi.org/10.1111/mafi.12170.

Bølviken, E., \& Guillen, M. (2017). Risk aggregation in Solvency II through recursive log-normals. Insurance: Mathematics and Economics, 73, 20-26. https://doi.org/10.1016/j.insmatheco.2016.12.006.

Boonen, T. J. (2019). Static and dynamic risk capital allocations with the euler rule. Journal of Risk, 22(1), 1-15. https://doi.org/10.21314/JOR.2019.420.

Boonen, T. J., Tsanakas, A., \& Wüthrich, M. V. (2017). Capital allocation for portfolios with non-linear risk aggregation. Insurance: Mathematics and Economics, 72, 95-106. https://doi.org/10.1016/j.insmatheco. 2016.11.003.

Buch, A., \& Dorfleitner, G. (2008). Coherent risk measures, coherent capital allocations and the gradient allocation principle. Insurance: Mathematics and Economics, 42(1), 235-242. https://doi.org/10.1016/ j.insmatheco.2007.02.006.

Buch, A., Dorfleitner, G., \& Wimmer, M. (2011). Risk capital allocation for RORAC optimization. Journal of Banking and Finance, 35(11), 3001-3009. https://doi.org/10.1016/j.jbankfin.2011.04.001.

Chen, X., Chong, W. F., Feng, R., \& Zhang, L. (2020). Pandemic risk management: Resources contingency planning and allocation. arXiv:2012.03200.

Corrigan, J., De Decker, J., Hoshino, T., van Delft, L., \& Verheugen, H. (2009). Aggregation of risks and allocation of capital (Tech. Rep.). Seattle, WA: Milliman.

Cossette, H., Landriault, D., Marceau, E., \& Moutanabbir, K. (2016). Moment-based approximation with mixed erlang distributions. Variance, 10(1), 161-182.

Denault, M. (2001). Coherent allocation of risk capital. Journal of Risk, 4(1), 1-34. https://doi.org/10.21314/ JOR.2001.053.

Dhaene, J., Goovaerts, M., Lundin, M., \& Vanduffel, S. (2005). Aggregating economic capital. Belgian Actuarial Bulletin, 5, 52-56.

Dhaene, J., Tsanakas, A., Valdez, E. A., \& Vanduffel, S. (2012). Optimal capital allocation principles. Journal of Risk and Insurance, 79(1), 1-28. https://doi.org/10.1111/j.1539-6975.2011.01408.x.

Di Lascio, F. M. L., Giammusso, D., \& Puccetti, G. (2018). A clustering approach and a rule of thumb for risk aggregation. Journal of Banking and Finance, 96, 236-248. https://doi.org/10.1016/j.jbankfin.2018.07. 002 .

Embrechts, P., Puccetti, G., \& Rüschendorf, L. (2013). Model uncertainty and VaR aggregation. Journal of Banking and Finance, 37(8), 2750-2764. https://doi.org/10.1016/j.jbankfin.2013.03.014.

Feng, R. (2018). An introduction to computational risk management of equity-linked insurance. Boca Raton: CRC Press.

Filipović, D. (2009). Multi-level risk aggregation. ASTIN Bulletin: The Journal of the International Actuarial Association, 39(2), 565-575. https://doi.org/10.2143/AST.39.2.2044648.

Furman, E., Hackmann, D., \& Kuznetsov, A. (2020). On log-normal convolutions: An analytical-numerical method with applications to economic capital determination. Insurance: Mathematics and Economics, 90, 120-134. https://doi.org/10.1016/j.insmatheco.2019.10.003.

Furman, E., Kye, Y., \& Su, J. (2020). A reconciliation of the top-down and bottom-up approaches to risk capital allocations: Proportional allocations revisited. North American Actuarial Journal. https://doi. org/10.1080/10920277.2020.1774781.

Furman, E., \& Zitikis, R. (2008a). Weighted premium calculation principles. Insurance: Mathematics and Economics, 42(1), 459-465. https://doi.org/10.1016/j.insmatheco.2007.10.006.

Furman, E., \& Zitikis, R. (2008b). Weighted risk capital allocations. Insurance: Mathematics and Economics, 43(2), 263-269. https://doi.org/10.1016/j.insmatheco.2008.07.003.

Gómez, F., \& Tang, Q. (2020). The gradient allocation principle based on the higher moment risk measure. Working paper.

Heilmann, W. R. (1989). Decision theoretic foundations of credibility theory. Insurance: Mathematics and Economics, 8(1), 77-95. https://doi.org/10.1016/0167-6687(89)90050-4.

Kalkbrener, M. (2005). An axiomatic approach to capital allocation. Mathematical Finance, 15(3), $425-437$. https://doi.org/10.1111/j.1467-9965.2005.00227.x.

Kang, W. Y., \& Poshakwale, S. (2019). A new approach to optimal capital allocation for RORAC maximization in banks. Journal of Banking and Finance, 106, 153-165. https://doi.org/10.1016/j.jbankfin.2019.06.006.

Kuritzkes, A., Schuermann, T., \& Weiner, S. M. (2003). Risk measurement, risk management, and capital adequacy in financial conglomerates. Brookings-Wharton Papers on Financial Services, 2003(1), 141193. 
Laeven, R. J. A., \& Goovaerts, M. (2004). An optimization approach to the dynamic allocation of economic capital. Insurance: Mathematics and Economics, 35(2), 299-319. https://doi.org/10.1016/j.insmatheco. 2004.04.002.

McNeil, A. J., Frey, R., \& Embrechts, P. (2015). Quantitative risk management: Concepts, techniques and tools (Revised ed.). Princeton: Princeton University Press.

Miles, J., Furman, E., \& Kuznetsov, A. (2020). Risk aggregation: A general approach via the class of generalized gamma convolutions. Variance 132 forthcoming.

Morone, M., Cornaglia, A., \& Mignola, G. (2007). Economic capital assessment via copulas: aggregation and allocation of different risk types. Working paper.

Panjer, H. H., \& Jing, J. (2001). Solvency and capital allocation. University of Waterloo, Institute of Insurance and Pension Research, Research Report, 01-14 (pp. 1-8).

Perli, R., \& Nayda, W. I. (2004). Economic and regulatory capital allocation for revolving retail exposures. Journal of Banking and Finance, 28(4), 789-809. https://doi.org/10.1016/j.jbankfin.2003.10.007.

Pfeifer, D., \& Strassburger, D. (2008). Solvency II: Stability problems with the SCR aggregation formula. Scandinavian Actuarial Journal, 2008(1), 61-77. https://doi.org/10.1080/03461230701766825.

Powers, M. R. (2007). Using Aumann-Shapley values to allocate insurance risk: The case of inhomogeneous losses. North American Actuarial Journal, 11(3), 113-127. https://doi.org/10.1080/10920277. 2007.10597470.

Resti, A., \& Sironi, A. (2007). Risk management and shareholders'value in banking: From risk measurement models to capital allocation policies. Hoboken: Wiley.

Sarabia, J. M., Gómez-Déniz, E., Prieto, F., \& Jordá, V. (2016). Risk aggregation in multivariate dependent pareto distributions. Insurance: Mathematics and Economics, 71, 154-163. https://doi.org/10.1016/j. insmatheco.2016.07.009.

Tasche, D. (1999). Risk contributions and performance measurement. Working paper. (Munich University of Technology)

Tsanakas, A., \& Barnett, C. (2003). Risk capital allocation and cooperative pricing of insurance liabilities. Insurance: Mathematics and Economics, 33(2), 239-254. https://doi.org/10.1016/S01676687(03)00137-9.

Zaks, Y., Frostig, E., \& Levikson, B. (2006). Optimal pricing of a heterogeneous portfolio for a given risk level. ASTIN Bulletin: The Journal of the International Actuarial Association, 36(1), 161-185. https:// doi.org/10.1017/S0515036100014446.

Publisher's Note Springer Nature remains neutral with regard to jurisdictional claims in published maps and institutional affiliations. 